\title{
Comparison of density estimation methods for astronomical datasets
}

\author{
B. J. Ferdosi ${ }^{1}$, H. Buddelmeijer ${ }^{2}$, S. C. Trager ${ }^{2}$, M. H. F. Wilkinson ${ }^{1}$, and J. B. T. M. Roerdink ${ }^{1}$ \\ 1 Johann Bernoulli Institute for Mathematics and Computer Science, University of Groningen, PO Box 407, \\ 9700 AK Groningen, The Netherlands \\ e-mail: [b.j.ferdosi;m.h.f.wilkinson; j.b.t.m.roerdink]@rug.nl \\ 2 Kapteyn Astronomical Institute, University of Groningen, PO Box 800, 9700 AV Groningen, The Netherlands \\ e-mail: [buddel; sctrager] aastro.rug.nl
}

Received 12 March 2011 / Accepted 2 May 2011

\begin{abstract}
Context. Galaxies are strongly influenced by their environment. Quantifying the galaxy density is a difficult but critical step in studying the properties of galaxies.

Aims. We aim to determine differences in density estimation methods and their applicability in astronomical problems. We study the performance of four density estimation techniques: $k$-nearest neighbors $(\mathrm{kNN})$, adaptive Gaussian kernel density estimation (DEDICA), a special case of adaptive Epanechnikov kernel density estimation (MBE), and the Delaunay tessellation field estimator (DTFE).

Methods. The density estimators are applied to six artificial datasets and on three astronomical datasets, the Millennium Simulation and two samples from the Sloan Digital Sky Survey. We compare the performance of the methods in two ways: first, by measuring the integrated squared error and Kullback-Leibler divergence of each of the methods with the parametric densities of the datasets (in case of the artificial datasets); second, by examining the applicability of the densities to study the properties of galaxies in relation to their environment (for the SDSS datasets).

Results. The adaptive kernel based methods, especially MBE, perform better than the other methods in terms of calculating the density properly and have stronger predictive power in astronomical use cases.

Conclusions. We recommend the modified Breiman estimator as a fast and reliable method to quantify the environment of galaxies.
\end{abstract}

Key words. methods: data analysis - methods: statistical - methods: miscellaneous

\section{Introduction}

Estimating densities in datasets is a critical first step in making progress in many areas of astronomy. For example, a galaxy's environment apparently plays an important role in its evolution, as seen in the morphology-density relation (e.g., Hubble \& Humason 1931; Dressler 1980) or the color-density and colorconcentration-density relations (e.g., Baldry et al. 2006). For these relations, a consistent, repeatable - and hopefully accurate - estimate of the local density of galaxies is an important datum. As another example, reconstruction of the large-scale structure of the Universe requires a proper estimation of the cosmic density field (e.g., Romano-Díaz \& van de Weygaert 2007). Even simulations require density estimation: smoothed particle hydrodynamics (SPH) is a method to create simulated astronomical data using astrophysical fluid dynamical computation (Gingold \& Monaghan 1977; Lucy 1977), in which kernel-based density estimation is used to solve the hydrodynamical equations. Density estimation is not only required for analyzing spatial domain structures but also for structures in other spaces, like finding bound structures in six-dimensional phase space in simulations of cosmic structure formation (Maciejewski et al. 2009) or in three-dimensional projections of phase space in simulations of the accretion of satellites by large galaxies (Helmi \& de Zeeuw 2000).

In the current work we are motivated by a desire to quantify the three-dimensional density distribution of galaxies in large surveys (like the Sloan Digital Sky Survey, York et al. 2000, hereafter SDSS) in order to study environmental effects on galaxy evolution. We are also interested in finding structures in higher-dimensional spaces, like six-dimensional phase space or even higher-dimensional spaces in large astronomical databases (such as the SDSS database itself). We are therefore interested in accurate and (computationally) efficient density estimators for astronomical datasets in multiple dimensions.

In this paper we investigate the performance of four density estimation methods:

- $k$-nearest neighbors ( $\mathrm{kNN})$;

- a 3D implementation of adaptive Gaussian kernel density estimation, called DEDICA (Pisani 1996);

- a modified version of the adaptive kernel density estimation of Breiman et al. (1977), called the modified Breiman estimator (MBE); and

- the Delaunay tessellation field estimator (DTFE: Schaap \& van de Weygaert 2000).

The first method is well-known to astronomers and involves determining densities by counting the number of nearby neighbors to a point under consideration. This method is typically used in studies of the morphology-density relation and other observational studies of the relation between environment and galaxy properties (e.g., Dressler 1980; Balogh et al. 2004; Baldry et al. 2006; Ball et al. 2008; Cowan \& Ivezic 2008; Deng et al. 2009, just to mention a few studies). The second and third methods are 
both adaptive-kernel density estimators, where a kernel whose size adapts to local conditions (usually isotropically), depending on some criteria set before or iteratively during the estimation process, is used to smooth the point distribution so that typical densities can be estimated. The fourth method, like the first, uses the positions of nearby neighbors to estimate local densities. We compare the methods using artificial datasets with known densities and three astronomical datasets, including the Millennium simulation of Springel et al. (2005) and two samples of real galaxies drawn from SDSS.

This paper is organized as follows. Section 2 discusses the four density estimation methods under consideration. Section 3 describes the datasets we used. Section 4 contains a comparison between the methods based on datasets with both known and unknown underlying density fields. Finally, in Sect. 5 we summarize our findings and draw conclusions.

We point out here that our goal here is not to quantify the shape of the environments of objects in datasets, but rather to estimate the density field or the densities at specific points in those datasets (see below). Information about the shapes of the structures found in the datasets is beyond the scope of this work; we refer the interested reader to recent excellent studies by, e.g., Jasche et al. (2010), Aragón-Calvo et al. (2010) and Sousbie et al. (2009).

\section{Density estimation methods}

The purpose of a density estimator is to approximate the true probability density function (pdf) of a random process from an observed dataset. There are two main families of density estimators: parametric and non-parametric. In parametric methods the type of distribution (uniform, normal, Poisson etc.) of the phenomenon needs to be known (or guessed) beforehand, whereas non-parametric methods do not need this information. The methods under consideration in this study belong to the second type.

First, though, we must distinguish different types of estimated densities. Starting from an input dataset consisting of a list of point positions $\boldsymbol{r}_{i} \in \mathbb{R}^{d}, i=1, \ldots, N$ in a $d$-dimensional spatial domain, we define two types of probability density as:

1. point probability densities: probability densities $\hat{p}\left(\boldsymbol{r}_{i}\right)$ at the original point positions $\boldsymbol{r}_{i}$;

2. probability density field: probability densities $\hat{p}(\boldsymbol{r})$ at arbitrary points in the spatial domain of $\mathbb{R}^{d}$. We often evaluate field densities at the points of a Cartesian $d$-dimensional grid and therefore also speak of grid densities.

Furthermore, the probability densities have to be converted to physical densities when comparing galaxies. This is because the parameter of interest is a quantification of the environment of individual galaxies, not the probability of finding a galaxy at a specific position. The latter is calculated by the density estimators and can be converted into the former by multiplying by $N$, i.e.,

1. point number densities: $\hat{\rho}\left(\boldsymbol{r}_{\boldsymbol{i}}\right)=N \hat{p}\left(\boldsymbol{r}_{\boldsymbol{i}}\right)$;

2. number density field: $\hat{\rho}(\boldsymbol{r})=N \hat{p}(\boldsymbol{r})$.

\section{1. k-nearest neighbor method}

The kNN estimator is well-known in astronomy and its working principle is to center a spherical window onto each point $\boldsymbol{r}$ and let it grow until it captures $k$ samples (the $k$ nearest-neighbors of $\boldsymbol{r}$ ). Then the kNN density estimate for a dataset with $N$ data points is defined at any $\boldsymbol{r} \in \mathbb{R}^{d}$ by

$\hat{p}(\boldsymbol{r})=\frac{1}{N} \frac{k}{V_{\mathrm{d}} \delta_{k}^{d}}$,

where $\delta_{k}$ is the distance of the $k$ th nearest neighbor from $\boldsymbol{r}$ and $V_{\mathrm{d}}$ the volume of the unit sphere in $d$-dimensional space. The kNN approach uses a different window size for each point so it adapts to the local density: when the density is high near $r$, the window will be small; but when the local density is low, the window will grow to a larger size.

The kNN approach can be a good solution for finding the "best" window size. However, this method suffers from a number of deficiencies. The resulting density estimate is not a proper probability density since its integral over all space diverges, and its tails fall off extremely slowly (Silverman 1986). The density field is very "spiky" and the estimated density is far from zero even in the case of large regions with no observed samples, due to the heavy tails. Furthermore, it yields discontinuities even when the underlying distributions are continuous (Breiman et al. 1977).

In astronomical work it is typically the case that the sample point is not considered to be its own neighbor (e.g., Dressler 1980; Baldry et al. 2006). This presents a conceptual problem, as the point density will then disagree with the field density at the location of a sample point. In our work we take the sample point to be its own first neighbor as in Silverman (1986), and we use the average of $\mathrm{kNN}$-estimated densities with $k=5$ and $k=6$ when computing either the point or grid densities. This is not precisely equivalent to the average $k=4$ and $k=5 \mathrm{kNN}$ density used in many astronomical papers (e.g., Baldry et al. 2006). While the $V$ in the denominator of Eq. (1) would be equal, the $k$ in the nominator is one higher in Silverman's definition.

\subsection{Adaptive Epanechnikov kernel density estimation}

Breiman et al. (1977) described a case of an adaptive (Gaussian) kernel approach. This method begins by computing the distance $\delta_{i, k}$ to the $k$ th nearest neighbor of each data point located at $\boldsymbol{r}_{i}$, just as in a kNN density estimator. Rather than using this distance to compute the kNN density estimate, it uses this to steer the local kernel size (also known as bandwidth) in an adaptive kernel density estimator or Parzen estimator (Parzen 1962). For a sample $D_{N}$ of $N$ points with position vectors $\boldsymbol{r}_{i} \in \mathbb{R}^{d}(i=1, \ldots, N)$ and kernel $K(\boldsymbol{r})$, the adaptive kernel density estimate $\hat{p}(\boldsymbol{r})$ is then given by:

$\hat{p}(\boldsymbol{r})=\frac{1}{N} \sum_{i=1}^{N}\left(\alpha_{k} \delta_{i, k}\right)^{-d} K\left(\frac{\boldsymbol{r}-\boldsymbol{r}_{i}}{\alpha_{k} \delta_{i, k}}\right)$.

In their simulations Breiman et al. (1977) used a symmetric Gaussian kernel. Here $k$ and $\alpha_{k}$ are still to be determined. For $k$ or $\alpha_{k}$ too small, the result will be noisy, whereas if $k$ and $\alpha_{k}$ are large we lose detail. The proper parameter values for $\sigma$ (width of the normal distribution), $k$ and $\alpha_{k}$ were determined by optimizing certain goodness-of-fit criteria (for details see Breiman et al. 1977).

Silverman (1986) argues that we can interpret this as using a "pilot estimate" of the density. We can understand this by observing from (1) that

$\hat{p}_{\mathrm{kNN}}\left(\boldsymbol{r}_{i}\right) \propto \delta_{i, k}^{-d}$.

Thus the bandwidth at each location is proportional to $\hat{p}_{\mathrm{kNN}}^{-1 / d}\left(\boldsymbol{r}_{i}\right)$. Thus, Breiman et al. (1977) implicitly use a kNN pilot density 


\section{B. J. Ferdosi et al.: Density estimation methods}

estimate to steer the final density estimate. The effect is that in low density regions $\delta_{i, k}$ will be large and the kernel will spread out; in high density regions the opposite occurs.

\subsubsection{Fundamentals of the modified Breiman estimator (MBE)}

The approach of Breiman et al. (1977) used for finding proper parameter values is computationally expensive, because they need to run the estimator numerous times to find the optimal parameters. This is even more costly because the kernel has infinite support. This means that each data point contributes to the density at every position, resulting in an $O\left(N^{2}\right)$ cost per parameter setting tested.

We want to apply the method for astronomical datasets that are very large in size ( $>50000$ data points) and dimension (from 10 to hundreds). For this reason we use a fast and scalable modification of Breiman's method along the lines of Wilkinson \& Meijer (1995). It was observed by Silverman (1986), that the implicit kNN pilot estimate could be replaced by a different estimate without significant change in quality. Therefore, Wilkinson \& Meijer (1995) used the kernel density estimator itself for the pilot. Furthermore they replaced the infinite support Gaussian kernel by the finite support Epanechnikov kernel, which increases computation speed significantly, and is optimal in the sense of minimal mean integrated square error (Epanechnikov 1969). To increase computational speed of the pilot estimate, the pilot density field is calculated on grid points first, after which the pilot density for each data point is obtained by multilinear interpolation. The method is also scalable: even when the number of data points grows very large, the computation time remains bounded by the number of grid points (Wilkinson \& Meijer 1995).

In the modified version Eq. (2) becomes

$\hat{p}(\boldsymbol{r})=\frac{1}{N} \sum_{i=1}^{N}\left(\sigma \lambda_{i}\right)^{-d} K_{\mathrm{e}}\left(\frac{\boldsymbol{r}-\boldsymbol{r}_{i}}{\sigma \lambda_{i}}\right)$

where $K_{\mathrm{e}}$ is the Epanechnikov kernel defined as

$K_{\mathrm{e}}(\boldsymbol{t})=\left\{\begin{array}{cl}\frac{d+2}{2 V_{d}}(1-\boldsymbol{t} . \boldsymbol{t}) & \text { if } \boldsymbol{t} \cdot \boldsymbol{t}<1 \\ 0 & \text { otherwise }\end{array}\right.$

in which $V_{d}$ is the volume of the unit sphere in $d$-dimensional space.

The density estimation proceeds in two phases.

Phase 1. Compute an optimal pilot window width $\sigma^{\text {opt }}$ with a percentile of the data as defined in Eq. (8) below. Define a pilot density $\hat{p}_{\text {pilot }}$ by using Eq. (4) with $\sigma=\sigma^{\text {opt }}$ and $\lambda_{i}=1$.

Phase 2 . From the pilot density $\hat{p}_{\text {pilot }}$ compute the local bandwidth parameters $\lambda_{i}$ by

$\lambda_{i}=\left(\frac{\hat{p}_{\text {pilot }}\left(\boldsymbol{r}_{i}\right)}{g}\right)^{-\alpha}$.

Here $g$ is the geometric mean of the pilot densities and $\alpha=$ $1 / d$ is the sensitivity parameter. The value of $1 / d$ is chosen to be equivalent to the method of Breiman et al. (1977), though some authors prefer a value of $1 / 2$ regardless of $d$ (Silverman 1986). The final density estimate is given by Eq. (4) once again, but now with $\sigma=\sigma^{\text {opt }}$ and $\lambda_{i}$ as given by Eq. (6).
Compared to the original method of Breiman et al., it should be noted that a fixed window width $\sigma^{\text {opt }}$ for the pilot estimate is used, rather than a fixed value of $k$. During the second phase of the algorithm we vary the window width with the density at each data point via the local bandwidth parameter. Data points with a low pilot estimate get a large window and vice versa.

\subsubsection{The pilot density estimate}

In the literature there exists a variety of methods to choose the optimal window width $\sigma^{\text {opt }}$ automatically. Basically there are two families of methods known: (i) classical (such as leastsquare cross-validation); and (ii) plug-in methods. In the latter case, the bias of an estimate $\hat{p}$ is written as a function of the unknown $p$, and usually approximated through Taylor series expansions. A pilot estimate of $p$ is then "plugged in" to derive an estimate of the bias (Loader 1999). However, there is some debate about the merits of these methods. For example, Park \& Marron (1990) found that the performance of least squares cross-validation is not very satisfactory. They recommended the plug-in methods for bandwidth selection. There are several other authors who have made strong comments about the classical approach and advocated plug-in methods (Ruppert et al. 1995; Sheather 1992). On the other hand, Loader (1999) strongly opposed these views. He argued that the plug-in methods can be criticized for the same reason the above authors criticized classical approaches.

We have already mentioned that the datasets that we will use are very large in size. Selecting bandwidth by cross-validation or a plug-in approach could consume more time than the density estimation itself. Therefore, we looked for simpler methods that can give an accurate estimate for the window width. Moreover, this window width is only used for the pilot estimate and for this purpose the desired window width should be large enough so that two consecutive window placements cover an overlapping area. For window width we tried max-min, percentile, median, standard deviation and average distance of the data points, normalized by the logarithm of the number of data points. We found that using percentile (Eq. (8)) as window width works well (in terms of the integrated squared error, see Sect. 2.5.1) even in the presence of outliers. However, the max-min window width works better if the dataset contains no outliers. Nevertheless, we recommend user interaction for changing the window width in the case of an under/oversmoothed density field.

Our procedure for the automatic determination $\sigma^{\text {opt }}$ can be summarized as follows. First window sizes $\sigma_{x}, \sigma_{y}, \sigma_{z}$ in each of the coordinate directions are computed by

$\sigma_{\ell}=\frac{P_{80}(\ell)-P_{20}(\ell)}{\log N}, \quad \ell=x, y, z$

where $P_{80}(\ell)$ and $P_{20}(\ell)$ are the 80th and 20th percentile of the data points in each dimension $\ell=x, y, z$. Then, in order to avoid oversmoothing, the optimal pilot window size $\sigma^{\text {opt }}$ is chosen as the smallest of these, i.e.,

$\sigma^{\mathrm{opt}}=\min \left\{\sigma_{x}, \sigma_{y}, \sigma_{z}\right\}$

\subsection{Adaptive Gaussian kernel density estimation (DEDICA)}

Pisani (1996) proposed a kernel-based density estimation method for multivariate data which is an extension of his work for the univariate case (Pisani 1993). Again this is an adaptive kernel estimator. The main differences with the MBE method 
are that a Gaussian kernel is used and that the optimal bandwidths are determined in an iterative way by minimizing a crossvalidation estimate. In our study, we use the 3D density estimator DEDICA, which is the FORTRAN implementation by Pisani.

\subsubsection{Fundamentals of the method}

For a sample $D_{N}$ of $N$ points with position vectors $\boldsymbol{r}_{i} \in \mathbb{R}^{d},(i=$ $1, \ldots, N)$ and kernel width of the $i$ th point given by $\sigma_{i}$, the adaptive Gaussian kernel density estimate $\hat{p}(\boldsymbol{r})$ is given by

$\hat{p}(\boldsymbol{r})=\frac{1}{N} \sum_{i=1}^{N} K_{n}\left(\left|\boldsymbol{r}_{i}-\boldsymbol{r}\right|, \sigma_{i}\right)$

where $K_{n}(t, \sigma)$ is the standard $d$-dimensional Gaussian kernel

$$
K_{n}(t, \sigma)=\frac{1}{\left(2 \pi \sigma^{2}\right)^{d / 2}} \exp \left[-\frac{t^{2}}{2 \sigma^{2}}\right] .
$$

The kernel widths $\sigma_{i}$ are chosen by an iterative method that minimizes the integrated square error locally. The procedure is as follows.

1. Initialize the window width:

$$
\sigma^{(0)}=4 \sigma_{t}, \quad \sigma_{t}=A(K) N^{-\frac{1}{d+4}} \sqrt{\frac{1}{d} \sum_{l=1}^{d} s_{l l}^{2}}
$$

where $s_{l l}$ is the standard deviation of the $l$ th coordinate of the data and $A(K)=0.96$ for a Gaussian kernel (Silverman 1986).

2. Iteratively perform the following steps for $n=1,2, \ldots$ :

(a) halve the window width: $\sigma^{(n)}=\sigma^{(n-1)} / 2$;

(b) compute a pilot estimate $\hat{p}_{\text {pilot }}^{(n)}\left(\boldsymbol{r}_{i}\right)$ by (9) with fixed kernel sizes $\sigma_{i}=\sigma^{(n)}$

(c) compute local bandwidth factors $\lambda_{i}^{(n)}$ by (6) with $\hat{p}_{\text {pilot }}=$ $\hat{p}_{\text {pilot }}^{(n)}$ and $\alpha=1 / 2$

(d) compute an adaptive kernel estimate $\hat{p}_{\mathrm{ka}}^{(n)}\left(\boldsymbol{r}_{i}\right)$ by (9) with adaptive kernel sizes $\sigma_{i}^{(n)}=\sigma^{(n)} \cdot \lambda_{i}^{(n)}$

(e) compute the cross-validation estimate (Pisani 1996, Eq. (7)):

$$
\begin{aligned}
M\left(\hat{p}_{\mathrm{ka}}^{(n)}\right)=\frac{1}{N^{2}} \sum_{i=1}^{N} \sum_{j=1}^{N} K_{n}\left(\left|\boldsymbol{r}_{i}-\boldsymbol{r}_{j}\right|,\left(\left(\sigma_{i}^{(n)}\right)^{2}+\left(\sigma_{j}^{(n)}\right)^{2}\right)^{\frac{1}{2}}\right) \\
-\frac{2}{N(N-1)} \sum_{i=1}^{N} \sum_{j \neq i} K_{n}\left(\left|\boldsymbol{r}_{i}-\boldsymbol{r}_{j}\right|, \sigma_{j}^{(n)}\right) .
\end{aligned}
$$

Minimization of the cross-validation estimate is equivalent to minimizing the integrated square error between the true density and the estimated density, see Pisani (1996) for more details.

3. Determine the iteration number $n=n_{\text {opt }}$ for which the crossvalidation estimate is minimized, and return the corresponding optimal window widths $\sigma_{i}^{\left(n_{\mathrm{opt}}\right)}$ and the adaptive kernel density estimate $\hat{p}_{\mathrm{ka}}^{\left(n_{\mathrm{opt}}\right)}\left(\boldsymbol{r}_{i}\right)$ at the sample points.

The cross-validation procedure can be understood by looking at the behaviour of the different terms in $M\left(\hat{p}_{\mathrm{ka}}^{(n)}\right)$. When $\sigma_{i}^{n}$ decreases during iteration, some terms will keep on increasing while others start to decrease when the local window sizes become much smaller than the inter-point distances. This is the point where the minimum of $M\left(\hat{p}_{\mathrm{ka}}^{(n)}\right)$ is reached and the iteration stops.

Although, as we will see below, DEDICA gives good results in many cases, it fails in certain situations. This can be attributed to some drawbacks of the method. First, the fixed kernel sizes $\sigma^{(n)}$ used for the pilot estimates form a discrete series of values (determined by the choice of $\sigma^{(0)}$ ). This series of values may be too coarse for finding the optimal window widths. Second, the method seeks a $\sigma_{i}^{\left(n_{\text {opt }}\right)}$ which leads to a globally optimal result, which, however, may be far from optimal in some regions.

We made an extension to the DEDICA code for obtaining the grid density, since the original code computes only point densities. We used the optimal window widths $\sigma_{i}^{\left(n_{\text {opt }}\right)}$ of each point calculated during the point density estimation to obtain the adaptive kernel density estimate $\hat{p}_{\mathrm{ka}}^{\left(n_{\mathrm{opt}}\right)}(\boldsymbol{r})$ at each grid point $\boldsymbol{r}$ by (9).

\subsection{Delaunay tessellation field estimator (DTFE)}

DTFE is a well-known method in astronomy to reconstruct density fields from a discrete set of scattered points (see, e.g., Schaap \& van de Weygaert 2000). In this method, the Delaunay tessellation (Okabe et al. 2000) of the points is constructed first. Then the point density is defined as the inverse of the total volume $V$ of the surrounding tetrahedra (in 3D) of each point, multiplied by a normalization constant (Schaap \& van de Weygaert 2000). For a sample $D_{N}$ of $N$ points with position vectors $\boldsymbol{r}_{i} \in$ $\mathbb{R}^{d},(i=1, \ldots, N)$, the DTFE density estimate $\hat{p}\left(\boldsymbol{r}_{i}\right)$ is given by:

$\hat{p}\left(\boldsymbol{r}_{i}\right)=\frac{1}{N} \frac{d+1}{V_{i}}$

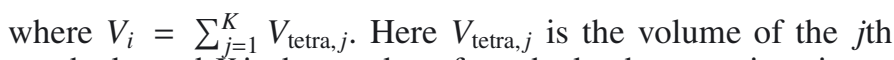
tetrahedra and $K$ is the number of tetrahedra that contain point $\boldsymbol{r}_{i}$.

In the next step, the density field is obtained by linearly interpolating the point densities $\hat{p}\left(\boldsymbol{r}_{i}\right)$ at the vertices of the Delaunay tetrahedra to the full sample volume.

\subsection{Error measures}

\subsubsection{Integrated squared error}

The integrated squared error (ISE) between the true density field and the density field obtained from each density estimator is one of our primary performance criteria in this study. The ISE is defined as:

$I S E=\int_{-\infty}^{\infty}(\hat{p}(\boldsymbol{r})-p(\boldsymbol{r}))^{2} \mathrm{~d} \boldsymbol{r}$

where $\hat{p}(\boldsymbol{r})$ is the estimated density and $p(\boldsymbol{r})$ is the true density.

\subsubsection{Generalized Kullback-Leibler divergence (Csiszar's I-divergence)}

Kullback-Leibler divergence (KLD) is one of the fundamental concepts in statistics that measures how far away a probability distribution $f$ is from another distribution $g$. It can also be interpreted in terms of the loss of power of the likelihood ratio test when the wrong distribution is used for one of the hypotheses (Eguchi \& Copas 2006). The value of $K L D(f, g)=$ 0 if $f=g$. However, the Kullback-Leibler divergence is only defined if $f$ and $g$ both integrate to 1 . Among the four methods under consideration, the density function estimated by kNN does not integrate to unity. Therefore, we use the generalized 
Table 1. Simulated datasets with known density distributions.

\begin{tabular}{|c|c|c|c|}
\hline Dataset & Component & Points & Distribution \\
\hline \multirow[t]{2}{*}{1} & Trivariate Gaussian 1 & 40000 & $C M_{1}=\operatorname{diag}(30)$ \\
\hline & Uniform random noise & 20000 & $\operatorname{Uniform}(x, y, z)=[0,100]$ \\
\hline \multirow[t]{3}{*}{2} & Trivariate Gaussian 1 & 20000 & $M_{1}=(25,25,25)$ \\
\hline & Trivariate Gaussian 2 & 20000 & $M_{2}=(65,65,65)$ \\
\hline & Uniform random noise & 20000 & $\operatorname{Uniform}(x, y, z)=[0,100]$ \\
\hline \multirow[t]{5}{*}{3} & Trivariate Gaussian 1 & 20000 & $M_{1}=(24,10,10)$ \\
\hline & Trivariate Gaussian 2 & 20000 & $M_{2}=(33,70,40)$ \\
\hline & Trivariate Gaussian 3 & 20000 & $M_{3}=(90,20,80)$ \\
\hline & Trivariate Gaussian 4 & 20000 & $M_{4}=(60,80,23)$ \\
\hline & Uniform random noise & 40000 & Uniform $(x, y, z)=[0,100]$ \\
\hline \multirow[t]{2}{*}{4} & Wall-like structure & 30000 & $\operatorname{Uniform}(x, y)=[0,100], \operatorname{Gaussian}(z)=[M=50, \operatorname{var}=5]$ \\
\hline & Filament-like structure & 30000 & $\operatorname{Uniform}(z)=[0,100], \operatorname{Gaussian}(x, y)=[M=50, \operatorname{var}=5]$ \\
\hline \multirow[t]{3}{*}{5} & Wall-like structure 1 & 20000 & $\operatorname{Uniform}(x, z)=[0,100], \operatorname{Gaussian}(\mathrm{y})=[M=10, \operatorname{var}=5]$ \\
\hline & Wall-like structure 2 & 20000 & $\operatorname{Uniform}(x, y)=[0,100], \operatorname{Gaussian}(z)=[M=50, \operatorname{var}=5]$ \\
\hline & Wall-like structure 3 & 20000 & $\operatorname{Uniform}(x, z)=[0,100], \operatorname{Gaussian}(y)=[M=50, \operatorname{var}=5]$ \\
\hline 6 & Log-normal & 60000 & $\log -\operatorname{normal}(x, y, z)=[M=3, \operatorname{var}=4]$ \\
\hline
\end{tabular}

Notes. $M=$ mean, $C M=$ covariance matrix.

Kullback-Leibler divergence (hereafter gKLD), also known as Csiszar's I-divergence (Csiszar 1991), to quantify the difference between two non-negative functions which have different integrals. For two positive functions $f(\boldsymbol{r})$ and $g(\boldsymbol{r})$, the gKLD is defined as:

$D(f \| g)=\int_{-\infty}^{\infty}\left(f(\boldsymbol{r}) \log \left(\frac{f(\boldsymbol{r})}{g(\boldsymbol{r})}\right)-f(\boldsymbol{r})+g(\boldsymbol{r})\right) \mathrm{d} \boldsymbol{r}$.

We compare the methods by comparing $D(p \| \hat{p})$.

Strictly speaking, the (generalized) Kullback-Leibler divergence is only defined when both true density $f(\boldsymbol{r})$ or the method density $g(\boldsymbol{r})$ are positive. This is a condition that is not fulfilled by our data: firstly, the boundary region of our "true" fields (approximately $23 \%$ of the total volume) has zero density; secondly, the DTFE and MBE methods produce density fields with zero values because they have finite support.

All methods except the DTFE estimate non-zeros for regions for which the true density is zero. This results in a gKLD value for $\mathrm{kNN}, \mathrm{MBE}$ and DEDICA that is lower than is justified: the discrepancy between the true and estimated field in this boundary region is not accounted for in the measure due to the multiplication by the true density $(f)$ in Eq. (15). The DTFE method behaves in the opposite way: it estimates zero densities where the true density is non-zero. We modified the gKLD such that if $g(\boldsymbol{r})=0$ we instead set $g(\boldsymbol{r})=\epsilon$, where $\epsilon$ is a small number. This results in a higher gKLD value for DTFE than is justified: the discrepancy in the boundary region can have a arbitrarily large effect (by choosing an arbitrarily low $\epsilon$ ) on the measure. However, we determined that this effect is small by comparing our gKLD value with the gKLD value calculated only over the regions where both fields are non-zero.

\section{Datasets}

We examined the performance of the four density estimation methods on three classes of datasets: a number of simulated datasets with known density fields to test the ability of each method to recover relatively simple density distributions; an astronomical dataset with an unknown but wellsampled density field based on the Millennium Simulation of Springel et al. (2005); and two different observed galaxy samples drawn from the Sloan Digital Sky Survey (SDSS: see, e.g., Adelman-McCarthy \& others 2007; Abazajian et al. 2009).

\subsection{Simulated datasets with known density fields}

We begin by constructing six simulated datasets with known density distributions (Table 1).

- Dataset 1 is a unimodal Gaussian distribution with added uniform noise.

- Dataset 2 contains two Gaussian distributions with an equal number of points but different covariance matrices (CMs) and different centers, again with added uniform noise; this dataset has the same number of points as Dataset 1 .

- Dataset 3 contains four Gaussian distributions with an equal number of points but different CMs and different centers, again with added uniform noise; this dataset has twice as many points as Datasets 1 and 2 .

- Dataset 4 contains a wall-like and a filament-like structure. The $x$ - and $y$-coordinates of the wall-like structure are drawn from a uniform distribution and the $z$-coordinate is drawn from a Gaussian distribution. The filament-like structure is created with a Gaussian distribution in the $x$ - and $y$-coordinates and a uniform distribution in $z$-coordinate.

- Dataset 5 contains three wall-like structures where each wall is created with a uniform distribution in two of the dimensions and a Gaussian distribution in the third.

- Dataset 6 contains points drawn from a lognormal distribution.

Scatter plot representations of these datasets are shown in Fig. 1.

The increasing complexity of these datasets allow us to probe simple situations ranging from idealized clusters to density fields that look somewhat like the large-scale structure of the Universe, with walls and filaments. The advantage of using simple simulations with known density distributions is clearly the ability to test the ability of the methods to recover the "true" point or field densities.

\subsection{Astronomical datasets with unknown density fields}

To test the performance of the methods on astronomical data we use three astronomical datasets: semi-analytic model galaxies 

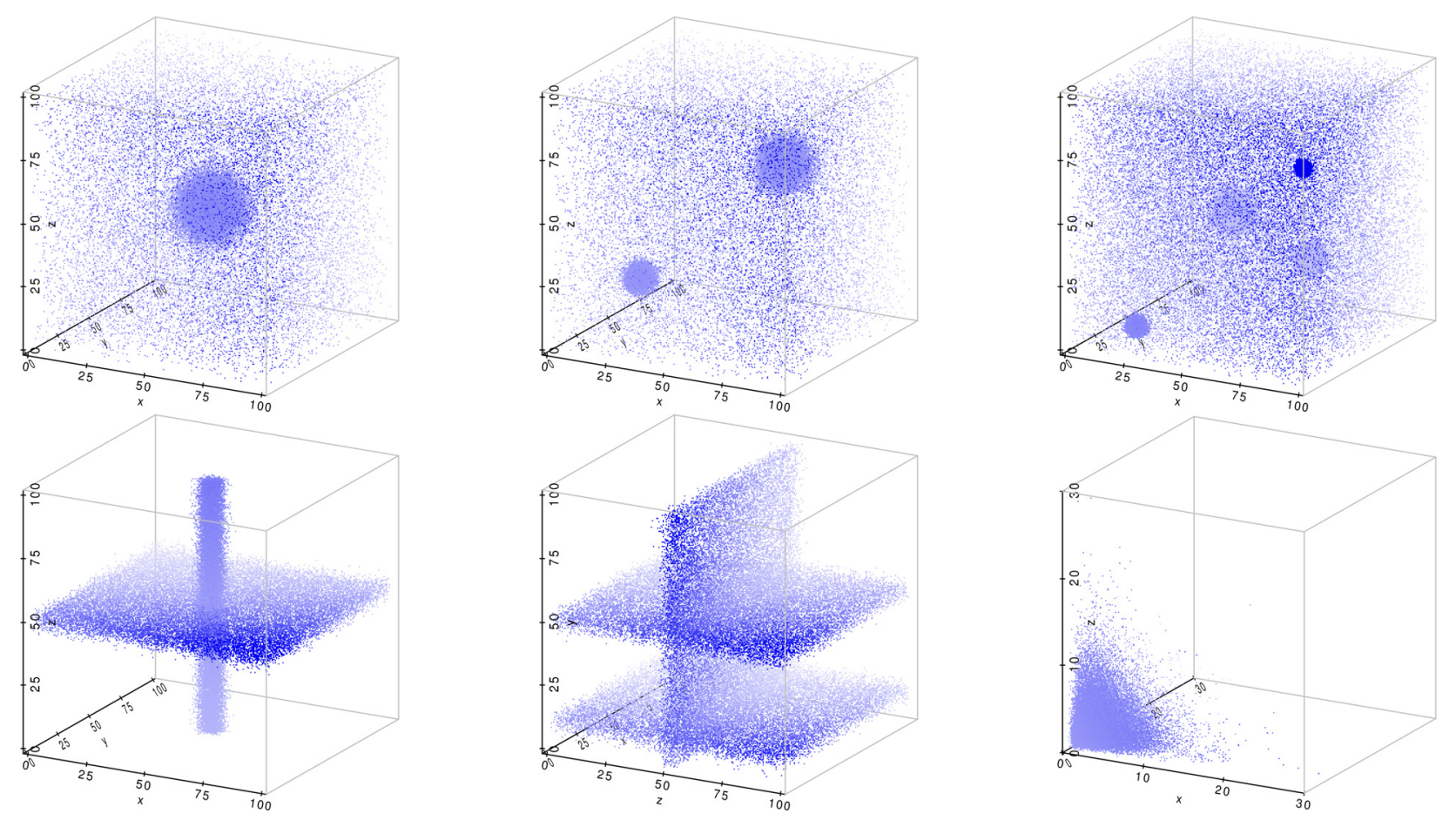

Fig. 1. Scatter plot representations of simulated datasets. Left to right, top to bottom: datasets 1-6.

drawn from the Millennium Simulation (Springel et al. 2005), and two samples of galaxies drawn from SDSS.

\subsubsection{The MSG dataset}

Our first astronomical dataset consists of the L-Galaxy sample of the "milliMil" subsample of the Millennium Simulation". The Millennium Simulation is one of the largest simulations ever to study the development of the Universe (Springel et al. 2005), following nearly $2 \times 10^{10}$ particles. It was created to make predictions about the large-scale structure of the universe and compare these against observational data and astrophysical theories. The L-Galaxies are created by populating halo trees drawn from the Millennium Simulation with semi-analytic models following the precepts in De Lucia \& Blaizot (2007). We use the much smaller "milliMillennium" ("milliMil") simulation, which sampled only $\sim 2 \times 10^{7}$ particles, and its associated L-Galaxies data. We refer to this dataset as the MSG dataset, which contains 53918 points. In a visual representation the output of the simulation looks like a fine three-dimensional web of filaments with fractal self-similarity and multiple layers of organization.

Our goal is to use the complexity of the MSG dataset to test the performance of the methods with a well-sampled but reasonably "astronomical" setting. Unfortunately, the true underlying density field of the MSG dataset is unknown. We therefore bootstrap MSG samples to define a "true density" for astronomical data. The density field of the MSG data is used to create new datasets and their density is taken to be the true density of those datasets. The process of creating new datasets can be described as follows:

Step 1: Calculate the density field of the MSG dataset using one of the density estimation methods.

\footnotetext{
${ }_{1}$ See http://www.g-vo.org/Millennium/Help?page=index
}

Step 2: Generate a new dataset by a Monte-Carlo process, which will have a probability density function similar to that of the MSG data, as follows:

1. generate a random ${ }^{2}$ position $r_{i}(x, y, z)$ within the original sample and a random value $p$ between zero and the maximum field density of the sample;

2. interpolate the density $P$ of a point $r_{i}(x, y, z)$ in the field obtained from step 1;

3. if $p<P$ accept the point $r_{i}(x, y, z)$ as a point in the new dataset; $P$ will be the "true" density of $r_{i}(x, y, z)$;

4. repeat step $2 \mathrm{a}-2 \mathrm{c}$ until the required number of points is obtained.

We generated two such datasets, one using DTFE (called the "MSG-DTFE" dataset) and another using MBE (called "MSGMBE"), each with the same number of points as the initial MSG dataset. For the MSG-MBE dataset the true density $P$ was interpolated from the grid of $256^{3}$ points and for the MSG-DTFE dataset from the Delaunay tessellation (see Appendix B). Scatter plot representations of these three fields - the original MSG dataset and the two derived datasets - are shown in Fig. 2. Note that both derived datasets look reassuringly like the original MSG dataset, although slight smoothing can been seen in both derived datasets.

Next, the field densities - on the grid - of the two new datasets generated by all density estimation methods are compared with the true densities obtained with the process described above.

\subsubsection{SDSS datasets}

Finally, to apply these density estimation methods to observed astronomical data we extract two galaxy samples from the

2 We used a random number generator based on the subtractive method of Knuth 1981 with a period of $2^{55}$. 

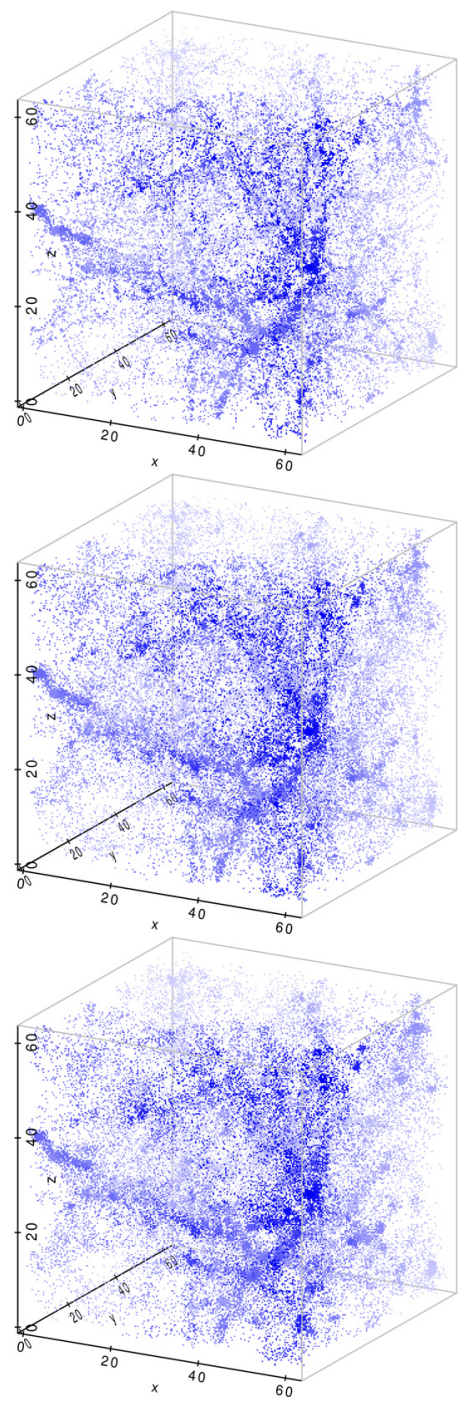

Fig. 2. Scatter plot representation of MSG and MSG-derived datasets. Top to bottom: MSG data, Dataset MSG-DTFE, Dataset MSG-MBE.

Seventh Data Release (DR7) of SDSS (Abazajian et al. 2009): a "cone" of galaxies over a relatively small solid angle on the sky but extended in redshift, and a " $z$-shell" of galaxies over a small redshift interval but a large solid area.

The spectroscopic redshift is used to calculate the comoving distance $R$ which is subsequently converted to Cartesian coordinates for density estimation, using a flat cosmology with $\Omega_{\mathrm{m}}=0.28, \Omega_{\Lambda}=0.72, h_{0}=0.7$.

\section{Completeness corrections}

A completeness correction is required when calculating densities from SDSS data, which we discuss before presenting the samples. SDSS is magnitude-selected but not (initially) constrained in redshift. This means that with distance, the number of galaxies in the sample drops because fainter galaxies can no longer be detected, causing underestimated densities for distant galaxies. To counter this effect, weights are calculated for every distance assuming a Schechter luminosity function (Schechter 1976; Felten 1977), following the procedure of Martínez \& Saar (2002). For this calculation all SDSS galaxies with spectroscopic distance between 50 and $2000 \mathrm{Mpc}$ (corresponding to redshifts from 0.0117 to 0.530 ) and Petrosian $r<17.7$ are used. If the galaxies
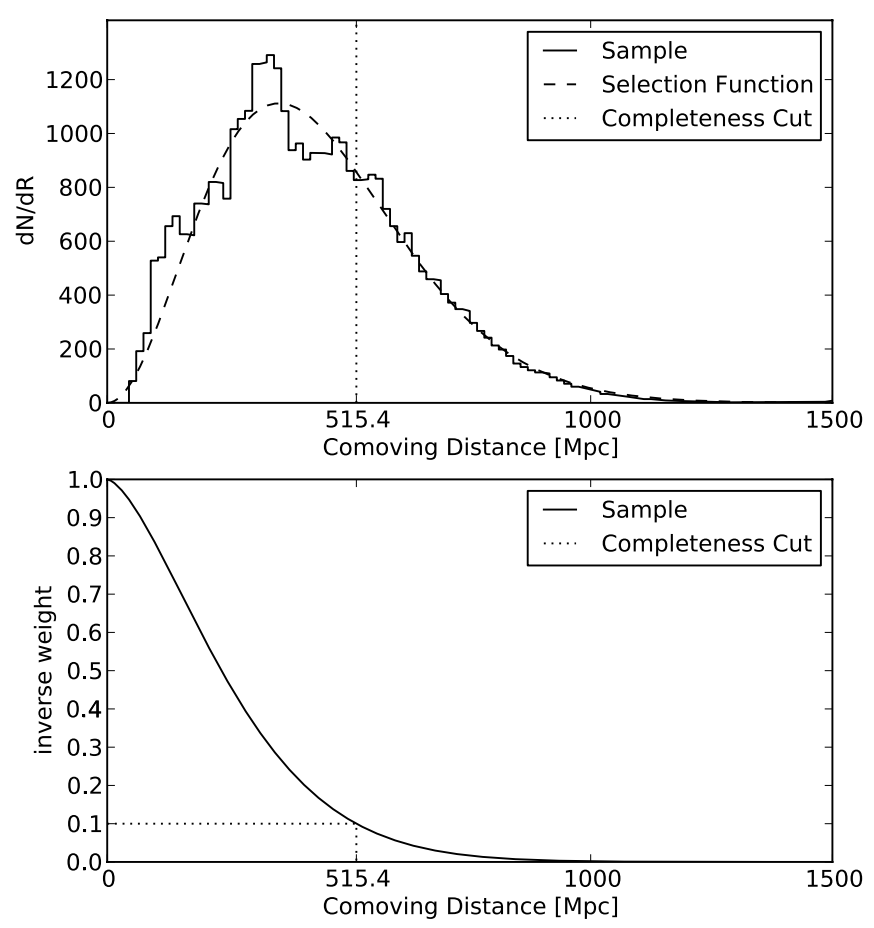

Fig. 3. Top: distance distribution of the SDSS spectroscopic legacy data in comoving distances assuming a concordance cosmology $\left(\Omega_{\mathrm{m}}=0.28\right.$, $\left.\Omega_{\Lambda}=0.72, h=0.7\right)$. The dashed line is a fit to this distribution assuming the galaxies follow a Schechter luminosity function, with an apparent magnitude limit of $r<17.7$ (see Eq. (16)). Bottom: the corresponding inverse weight as derived from the luminosity function. A $10 \%$ completeness level (corresponding to $R=515 \mathrm{Mpc}$, equivalent to $z=0.123$ ) is chosen to remove high redshift outliers.

follow a Schechter luminosity function, they should also follow a number distribution

$$
\frac{\mathrm{d} N}{\mathrm{~d} R}=\langle\rho(\boldsymbol{r})\rangle \Omega R^{2} \Phi(R)
$$

where $\langle\rho(\boldsymbol{r})\rangle$ is the average field density, $\Omega$ the survey area and $\Phi(R)$ is the selection function given by

$\Phi(R)=\mathrm{e}^{-\left(\frac{R}{R_{\mathrm{c}}}\right)^{\beta}}$.

The best fit of Eq. (16) to our data ( $\Omega=2.447 \mathrm{sr}$ ) is given by $\langle\rho(\boldsymbol{r})\rangle=0.013 \mathrm{Mpc}^{-3}, R_{\mathrm{c}}=299.8 \mathrm{Mpc}$ and $\beta=1.5$ and is shown in Fig. 3, top. The corresponding selection function is shown in Fig. 3, bottom. After calculation, the densities are corrected by dividing by the value of the selection function at the distance of the galaxy.

We note that due to the fiber masks used for the spectroscopy of SDSS, not all (bright) sources in dense environments have spectroscopic redshifts. These sources are not included in our sample, and we have not corrected for this, resulting in a bias of underestimated densities in the densest regions.

\section{The "cone" sample}

We choose 1939 "primary" galaxies within the rectangular boundary RA $=(185,190)$ and Dec $=(9,12)$ and with Petrosian $r<17.7$ and that have spectroscopic redshifts. The sky coverage of our sample is $14.7 \square^{\circ}$. 

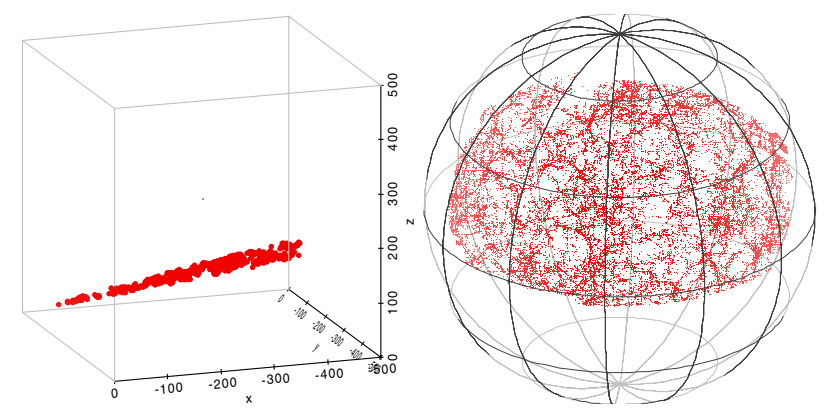

Fig. 4. Left: the cone sample, a $15 \square^{\circ}$ area within a redshift range of $z<0.123(R<515 \mathrm{Mpc})$. Right: the shell sample, selected from SDSS Northern Galactic Cap over the redshift range $0.10<z<0.11$, corresponding to a distance range of 418-459 Mpc.

A lower completeness limit (Fig. 3) of $10 \%$ is chosen to truncate the galaxy sample to limit the effect of high distance outliers; an incompleteness up to $90 \%$ does not cause unacceptably large errors when attempting to estimate the density of galaxies (see Appendix A). This corresponds to a distance of $R_{\text {max }}=515 \mathrm{Mpc}$ (redshift 0.123 ).

To prevent edge effects and to limit the effects of local motion, a lower limit for the distance is set at $R_{\min }=50.0 \mathrm{Mpc}$ (corresponding to a redshift of 0.0117 ). This results in a final number of galaxies in the "cone" sample of 1030. Volume densities were calculated using this magnitude- and redshift-limited sample of 1030 galaxies.

From integration of Eq. (16) for our cone sample $\left(\Omega_{\text {cone }}=\right.$ $0.00449 \mathrm{sr}$ ), it is expected that there are 2702 sources in the region of which we would detect 692. Instead, the cone sample has 1030 galaxies, $49 \%$ more than expected. Comparing with other regions of the same size shows that our cone sample is indeed extraordinary dense: out of the 24 other regions, only one had more sources than ours. Therefore we correct the average field density of the "cone" sample to $\left\langle\rho_{\text {cone }}(\boldsymbol{r})\right\rangle=0.0196 \mathrm{Mpc}^{-3}$.

The definition of $\sigma^{\text {opt }}$ for the MBE in Eq. (8) does not suffice for narrow cone-like samples. Problematic cases for such samples are a strong alignment with one axes (or planes) of the Cartesian coordinate system (our case), or an alignment with one of the space diagonals. The former results in a too-small $\sigma^{\text {opt }}$ value because one or two of the $\sigma_{l}$ values will be much smaller than the other(s), while the latter results in a too-high $\sigma^{\text {opt }}$ because $N$ (in the denominator of Eq. (7)) does not reflect the incomplete filling of space by the sample. Therefore we created a new definition of $\sigma^{\text {opt }}$ for conical samples: first the average distance of the nearest half of the galaxies is determined; then $\sigma^{\text {opt }}$ is chosen as the square root of the cross section of the cone at that distance.

We explore the effect of the "cone" sample selection on the performance of the density estimators in Appendix A.

\section{The "shell" sample}

To avoid the complication of the changing luminosity limit on the inferred densities, we also selected galaxies from SDSS in a thin shell in redshift space. For this "shell" sample, we choose 34558 "primary" galaxies in the Northern Galactic Cap (Abazajian et al. 2009) with redshifts in the range $0.10<z<$ 0.11 and a Petrosian magnitudes $r<17.7$ (Fig. 4).

To compare with the "cone" sample, the incompleteness correction is applied to the shell sample as well, enhancing the estimated densities by a factor of 5.3 to 6.9 .

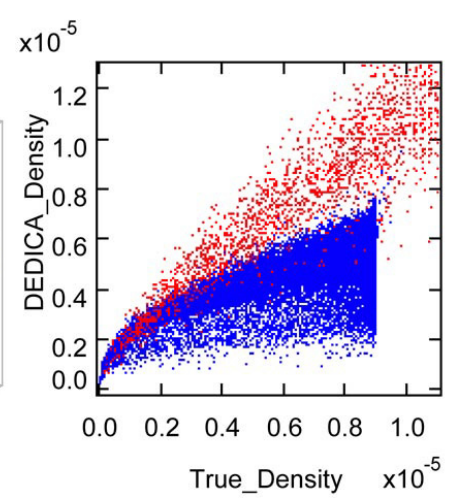

Fig. 5. Performance of DEDICA for dataset 4. Filament in red and the wall in blue. Left: spatial representation of the dataset. Right: comparison of true and DEDICA-inferred densities.

\section{Results}

We begin by examining the performance of the four density estimation methods on simulated datasets with known density fields. We find that the adaptive-kernel-based methods, MBE and DEDICA, best recover the input density distributions in these cases. We conclude this section by applying the density estimation methods to the SDSS samples and examine their utility for determining the color-density and color-concentration-density relations.

\subsection{Simulated datasets}

We first examine the performance of the four density estimation methods on the six simulated datasets and then on the two MSGderived datasets.

\subsubsection{Artificial datasets}

We compare the performance of the methods for the artificial datasets in the top rows of Table 2 using the ISE and the gKLD metrics. The true densities are parametric densities calculated using the parameters with which the datasets are created. It is clear that the adaptive-kernel-based methods, MBE and DEDICA, perform significantly better than $\mathrm{kNN}$ or DTFE in recovering the input density distributions. For all but Dataset 6, the lognormal distribution, the performance of $\mathrm{MBE}$ is better than or roughly equal to that of DEDICA. We note that the MBE densities were calculated with the automatic choice of the kernel size, and better performance of MBE might be obtained by modifying the smoothing parameter manually.

We note also that DEDICA performs very poorly for Dataset 4 (wall plus filament), where it fails to estimate the proper density. Examining the point densities in Fig. 5, it is clear that DEDICA underestimates the densities in the wall. We attribute this to the method failing to choose the proper kernel size during the automatic (cross-validation) kernel size selection on this dataset. We also see similar behavior when considering the MSG and SDSS datasets. We discuss this issue in more detail in Sect. 5.3.

Furthermore we note that the field produced by $\mathrm{kNN}$ is not normalized. For datasets 1 to 6 , the fields are approximately 25 to $30 \%$ over-dense on average. This is part of the reason that kNN performs the worst in terms of the integrated square error on these datasets. 
Table 2. Performance of density estimators: simulated and MSG datasets.

\begin{tabular}{|c|c|c|c|c|c|c|c|c|}
\hline \multirow[b]{2}{*}{ Dataset } & \multicolumn{4}{|c|}{ Integrated squared error } & \multicolumn{4}{|c|}{ Generalized Kullback-Leibler divergence } \\
\hline & MBE & DEDICA & DTFE & $\mathrm{kNN}$ & MBE & DEDICA & DTFE & $\mathrm{kNN}$ \\
\hline 1 & $2.23 \times 10^{-7}$ & $6.44 \times 10^{-6}$ & $1.54 \times 10^{-5}$ & $2.82 \times 10^{-5}$ & $5.61 \times 10^{-2}$ & $7.62 \times 10^{-2}$ & $1.83 \times 10^{-1}$ & $1.59 \times 10^{-1}$ \\
\hline 2 & $3.04 \times 10^{-6}$ & $1.75 \times 10^{-6}$ & $5.85 \times 10^{-5}$ & $1.19 \times 10^{-4}$ & $4.53 \times 10^{-2}$ & $8.34 \times 10^{-2}$ & $1.90 \times 10^{-1}$ & $1.62 \times 10^{-1}$ \\
\hline 3 & $4.74 \times 10^{-6}$ & $9.10 \times 10^{-6}$ & $1.99 \times 10^{-4}$ & $4.28 \times 10^{-4}$ & $3.90 \times 10^{-2}$ & $6.77 \times 10^{-2}$ & $1.62 \times 10^{-1}$ & $1.54 \times 10^{-1}$ \\
\hline 4 & $2.35 \times 10^{-6}$ & $2.91 \times 10^{-4}$ & $1.12 \times 10^{-5}$ & $2.02 \times 10^{-5}$ & $6.22 \times 10^{-2}$ & $1.33 \times 10^{+1}$ & $2.34 \times 10^{-1}$ & $1.79 \times 10^{-1}$ \\
\hline 5 & $5.65 \times 10^{-7}$ & $5.38 \times 10^{-7}$ & $1.31 \times 10^{-6}$ & $2.13 \times 10^{-6}$ & $1.01 \times 10^{-1}$ & $9.12 \times 10^{-2}$ & $2.42 \times 10^{-1}$ & $2.12 \times 10^{-1}$ \\
\hline 6 & $7.66 \times 10^{-4}$ & $7.94 \times 10^{-5}$ & $1.96 \times 10^{-3}$ & $3.71 \times 10^{-3}$ & $3.21 \times 10^{-1}$ & $6.32 \times 10^{-2}$ & $1.07 \times 10^{-1}$ & $1.43 \times 10^{-1}$ \\
\hline & $1.68 \times 10^{-3}$ & $4.86 \times 10^{-3}$ & $1.24 \times 10^{-3}$ & $1.39 \times 10^{-3}$ & $6.50 \times 10^{-1}$ & $2.18 \times 10^{+1}$ & $5.74 \times 10^{-1}$ & $5.73 \times 10^{-1}$ \\
\hline MSG-MBE & $6.89 \times 10^{-7}$ & $5.88 \times 10^{-4}$ & $1.95 \times 10^{-1}$ & $1.71 \times 10^{-4}$ & $3.00 \times 10^{-2}$ & $2.26 \times 10^{+1}$ & $1.25 \times 10^{0}$ & $3.08 \times 10^{-1}$ \\
\hline
\end{tabular}

Notes. Entries highlighted in boldface represent the smallest ISE or gKLD value and therefore the "best" method for that dataset under that performance metric.

\subsubsection{The MSG datasets}

We compare the performance of the density estimators on the MSG datasets in the bottom rows of Table 2. As expected, DTFE performs best on the MSG-DTFE dataset, and MBE performs best on the MSG-MBE dataset. Interestingly, $\mathrm{kNN}$ performs as well as DTFE on the MSG-DTFE dataset. This is not a complete surprise, as DTFE and kNN are conceptually similar, because both use only points in the immediate vicinity of the current location to estimate the density directly. Because of this, both may perform better than kernel estimates in the presence of strong gradients or even discontinuities in the underlying density. Despite this, MBE performs nearly as well as DTFE and kNN on the MSG-DTFE dataset, suggesting that MBE continues to perform well even on spatially-complex datasets.

The gKLD metric in Table 2 reveals that DEDICA fails to estimate proper densities for the samples from the Millennium dataset. For both MSG samples, DEDICA produces very different density distributions when compared with the "true" distribution (see the MSG-MBE dataset Fig. 6). As noted above, we observed a similar performance of DEDICA on the simulated Dataset 4, which contains a filament-like structure. The MSG dataset also contains obvious filamentary structure. Again, it appears that the automatic kernel size selection (using crossvalidation) of DEDICA failed to choose proper kernel size for such datasets (although it performs quite well in Gaussian and lognormal cases). We summarize this issue in Sect. 5.3.

\subsection{Application to SDSS datasets}

We now examine the application of our density estimators to the two observed galaxy datasets from SDSS, the "cone" and "shell" samples defined in Sect. 3.2.2 above.

\subsubsection{Density magnitude distributions}

We begin by comparing the distributions of the values of the densities [recall that $\hat{\rho}\left(\boldsymbol{r}_{\boldsymbol{i}}\right)=N \hat{p}\left(\boldsymbol{r}_{i}\right)$ ] produced by the four different methods (Fig. 7). (Note that in this subsection "density distribution" refers to the 1-D distribution of the magnitude of the density, not to the density distribution in space.) All four density estimation methods produce approximately lognormal distributions of the values $\hat{\rho}\left(\boldsymbol{r}_{i}\right)$ for the SDSS samples (as expected from previous studies and theoretical ideas: see, e.g., Coles \& Jones 1991). Therefore our analysis is performed with the logarithm of the density $\rho_{l}=\log _{10}\left(\hat{\rho}\left(\boldsymbol{r}_{i}\right)\right)$ or else a "standardized density" defined as

$\rho_{\mathrm{s}}=\frac{\rho_{l}-\mu_{l}}{\sigma_{l}}$,

where $\mu_{l}$ and $\sigma_{l}$ are the mean and standard deviation of the (almost) Gaussian density distributions. We plot the logarithmic density distributions in Fig. 7.

The true mean density of galaxies $\langle\rho(\boldsymbol{r})\rangle$ for the "cone" and "shell" samples is respectively 0.0196 and 0.013 galaxies per cubic megaparsec (Sect. 3.2.2). The mean of the estimated densities $\left\langle\hat{\rho}\left(\boldsymbol{r}_{i}\right)\right\rangle$ cannot directly be compared against this number, since $\left\langle\hat{\rho}\left(\boldsymbol{r}_{\boldsymbol{i}}\right)\right\rangle$ is averaged over the set of galaxies and $\langle\rho(\boldsymbol{r})\rangle$ over the field. High density regions contain more galaxies and therefore have a heavier weight in the mean of the point densities $\left\langle\hat{\rho}\left(\boldsymbol{r}_{\boldsymbol{i}}\right)\right\rangle$. This weight is proportional to the density and if a lognormal distribution of the estimated densities is assumed, the mean of the estimated field densities $\langle\hat{\rho}(\boldsymbol{r})\rangle$ can be calculated as

$\langle\hat{\rho}(\boldsymbol{r})\rangle=\mathrm{e}^{\ln 10 \mu_{l}-\frac{\left(\ln 10 \sigma_{l}\right)^{2}}{2}}$.

For each estimator, the calculated value of $\langle\hat{\rho}(\boldsymbol{r})\rangle$ is plotted in Fig. 7 as well as the known average field density. For the "cone" sample, DTFE best approximates the known field average density, closely followed by MBE. For the "shell" sample this order is reversed. DEDICA does not correctly represent the known field average density and $\mathrm{kNN}$ is in between.

The distributions of the "shell" sample are smoother than those of the "cone" sample, due to the higher number of data points. Even for the "shell" sample, the DEDICA density distribution is not smooth, due to its global optimization nature that leads to tiny window widths (see Sect. 5.3). The MBE density distribution peaks at slightly higher densities for the "shell" sample. Apart from the difference in means and widths, the differences of the density methods manifest themselves in the tails of the estimated density distribution. DTFE produces high-density tails, as it is sensitive to overdensities due to the local nature of the method. MBE produces a low-density tail. The distribution from kNN both has stronger high- and low-end tails (compared to a Gaussian).

The density distribution of DEDICA is offset from the other distributions. By comparing the estimated field average density and the true field average density it is clear that the calculated values cannot represent the actual densities. This is due to the sensitivity of DEDICA to overdensities: in case of highly clustered data such as ours, it creates very small kernels, undersmoothing the density field (see Sect. 5.3). Moving the positions of the galaxies by $1 \mathrm{Mpc}$ in a random direction, thereby homogenizing the sample a little, removes this effect almost entirely. 

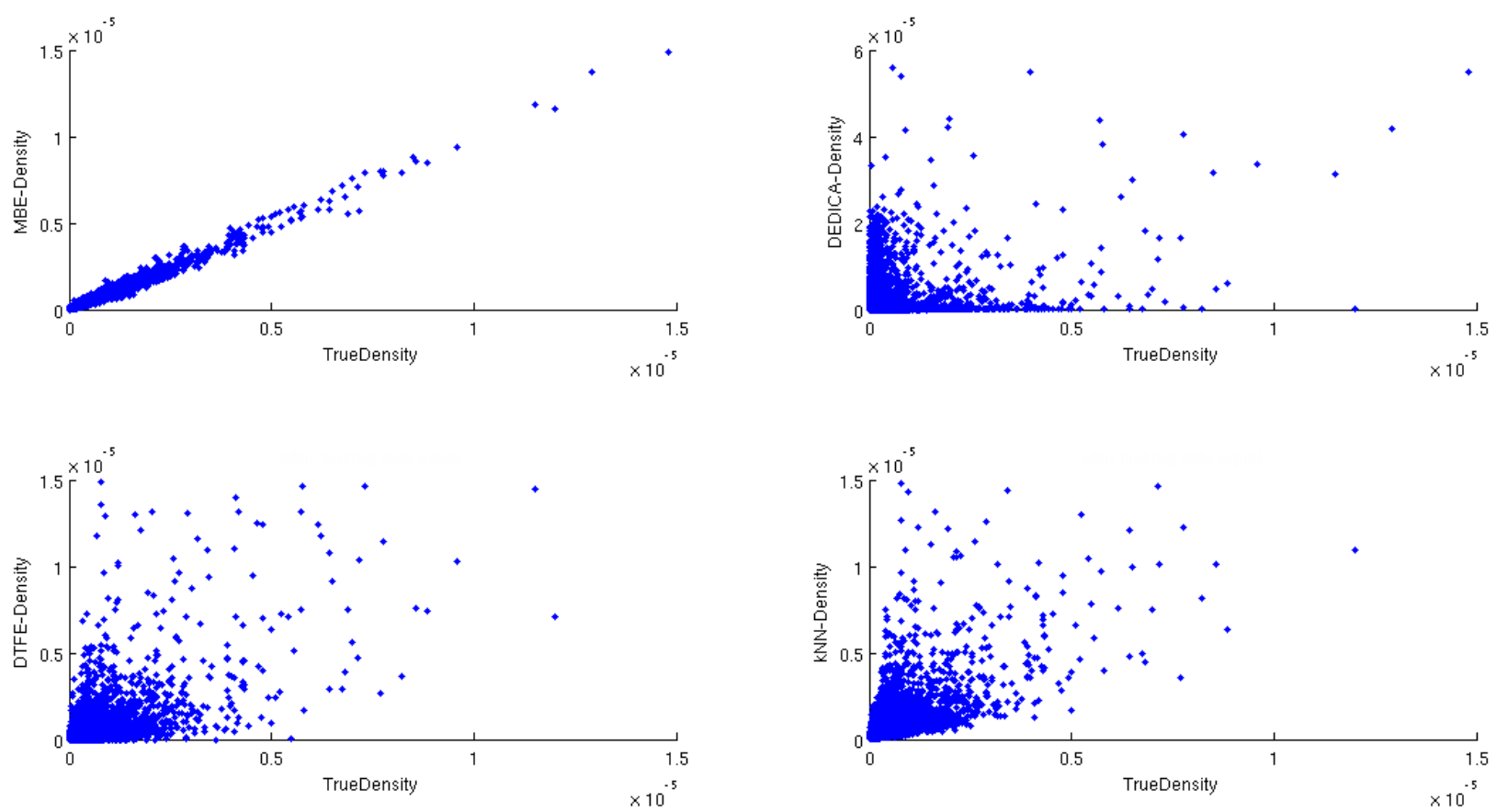

Fig. 6. Plot of true versus estimated field densities of the MSG-MBE dataset by MBE (top left), DEDICA (top right), DTFE (bottom left) and kNN (bottom right). Approximately 16000 random grid locations are shown.
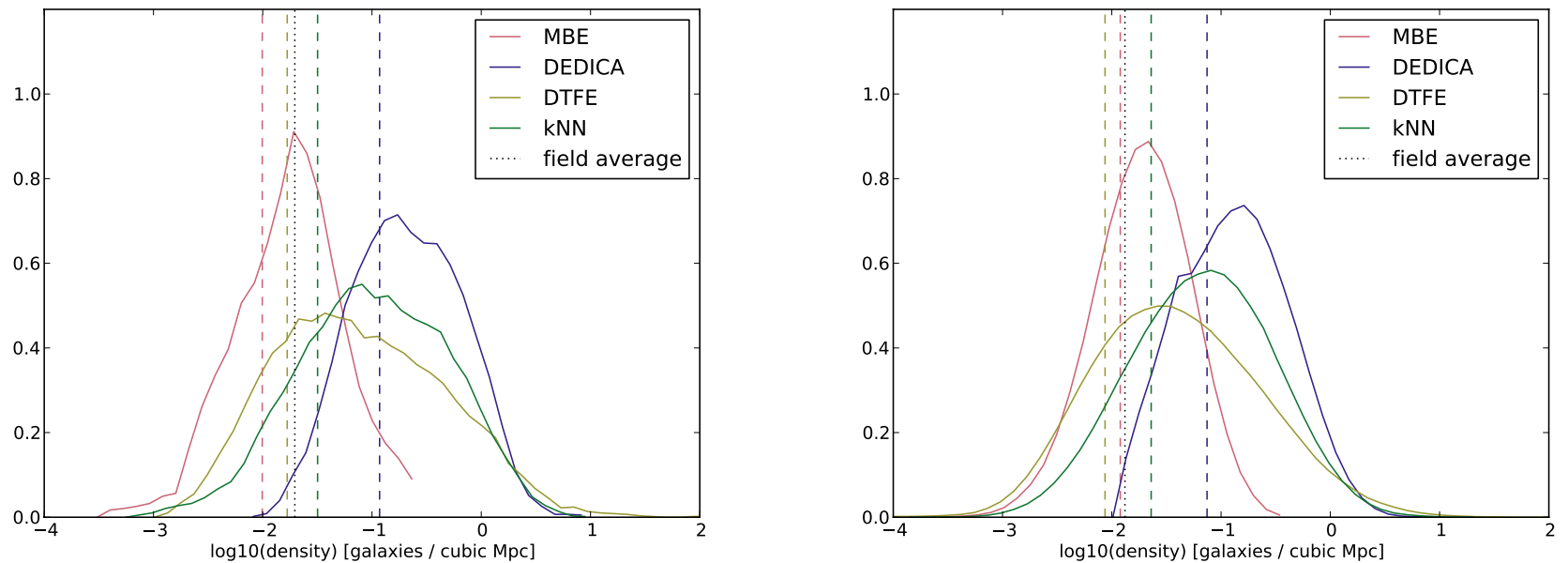

Fig. 7. The normalized distribution of the density values in log-space for each estimator. The distributions are smooth and close to Gaussian. The average field densities as calculated with Eq. (19) are plotted as dashed lines. A broader range in densities (DTFE, kNN) denotes that the estimator detects more clustering. More clustering results in more galaxies in higher density regions, shifting the peak of the distribution to the right. The dotted line represents the measured average field density from the selection function (see text). Left: "cone" sample. Right: "shell" sample.

However, even though the densities of the DEDICA galaxies are much higher than is expected, it can still be used as a parameter describing the environment of the galaxies by using it in standardized form.

\subsubsection{Galaxy color and concentration as a function of environmental density}

Two applications of the estimated densities are the exploration of morphology-density relation (see, e.g., Dressler 1980;
Baldry et al. 2006, in the context of the concentration-density relation) and environmental effects on the color-magnitude relation (e.g., Balogh et al. 2004; Baldry et al. 2006; Ball et al. 2008). We define the inverse concentration index as

$i C=\frac{r_{50}}{r_{90}}$,

where $r_{50}$ and $r_{90}$ are the radii containing $50 \%$ and $90 \%$ of the Petrosian flux (Baldry et al. 2006). For each galaxy, $i C$ is taken as the average of this ratio in the $r$ and $i$ bands. For typical galaxies, the inverse concentration ranges from 0.3 (concentrated) to 


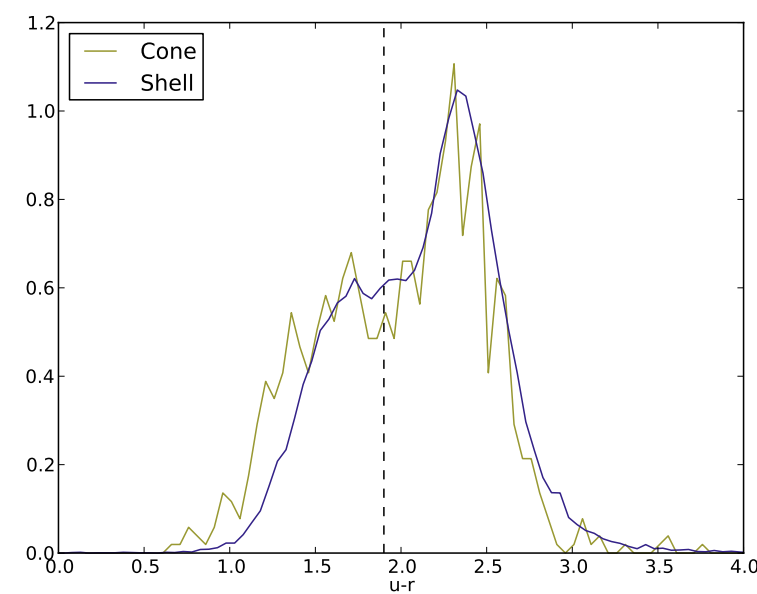

Fig. 8. The color distribution of the SDSS samples. The dotted line is the division between blue $(u-r<1.9)$ and red $(u-r \geq 1.9)$ galaxies.

0.55 (extended). A uniform disc would have $i C=0.75$. Galaxy colors are computed as the difference between absolute magnitudes after $k$-correction ${ }^{3}$ and extinction corrections.

It has been long known that the distribution of galaxy colors is bimodal, with blue galaxies being dominantly extended and disk-like and red galaxies being mostly compact and spheroidal (at least in the local Universe: see, e.g., Strateva et al. 2001, for a recent restatement of this observation). We show the color distributions of the two SDSS samples and our selected cut between blue and red galaxies in Fig. 8.

\subsubsection{The color-density relation}

As discussed in the introduction, "early-type" red galaxies are far more common in clusters of galaxies than in the general, low-density field, which is populated mostly by "late-type" blue galaxies (see, e.g., Hubble \& Humason 1931; Dressler 1980; Balogh et al. 2004; Baldry et al. 2006).

We compare the ability of the density estimators to recover the existence of this relation. We examine the galaxy colors in our "cone" SDSS samples as a function of environmental density parametrized as the "standardized" density defined above. The standardized density is binned in ten steps of $0.25 \sigma$ from the mean, resulting in 20 bins. The distribution for the counted numbers of red $\left(N_{\mathrm{r}}\right)$ and blue $\left(N_{\mathrm{b}}\right)$ galaxies in each bin is Poissonian around the respective means $\mu_{\mathrm{r}}$ and $\mu_{\mathrm{b}}$,

$P(N \mid \mu)=\frac{\mathrm{e}^{-\mu} \mu^{N}}{N !}$

The parameters of interest are $\mu_{\mathrm{r}}$ and $\mu_{\mathrm{b}}$, the distributions of which are also given by a Poissonian distribution,

$P(\mu \mid N)=\frac{\mathrm{e}^{-\mu} \mu^{N}}{N !}$

The fraction $(f)$ of red galaxies relative to the total number of galaxies is

$f=\frac{\mu_{\mathrm{r}}}{\mu_{\mathrm{r}}+\mu_{\mathrm{b}}}$.

\footnotetext{
$3 k$-corrections are calculated with KCORRECT v4.1.4 (Blanton \& Roweis 2007) using the Petrosian apparent magnitudes and spectroscopic redshifts.
}
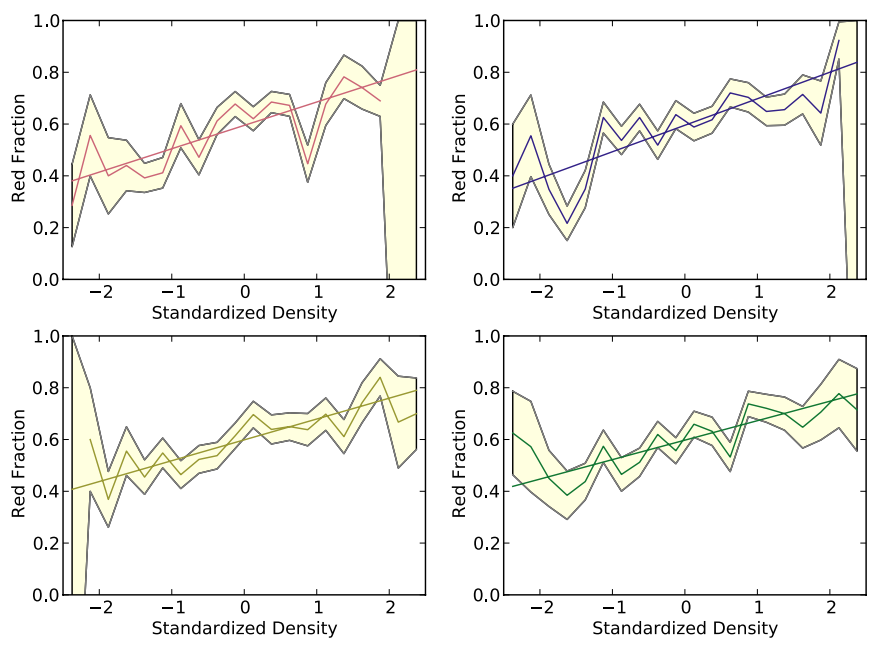

Fig. 9. Fraction of red galaxies as a function of standardized density. In reading order: MBE, DEDICA, DTFE, $\mathrm{kNN}$. The data is binned in 20 bins of width $0.25 \sigma$ centered around the mean. The yellow region denotes the error in the calculated red fraction as determined from the Monte Carlo simulation. In all cases, a clear color-density relation can be seen. The MBE shows a clear dip in high density regions. DEDICA has such a dip in low density regions.

A Monte Carlo process is used to estimate the $68 \%$ confidence intervals for the expected value of $f$ for every bin. To model this fraction as function of the standardized density $\rho_{\mathrm{s}}$, a straight line parametrized as

$f_{\text {model }}=a \rho_{\mathrm{s}}+c$

is fit to the data. Bins without either red or blue galaxies are given a zero weight so they do not contribute to the fit. The degrees of freedom (d.o.f.) are the number of bins that contain red and blue galaxies minus two, since the fitted model has two parameters.

Figure 9 shows the fraction of red galaxies of the "cone" sample as a function of standardized densities and the best-fitting straight lines. All estimators consistently find $c=0.60$ within one standard deviation of $\sigma_{\mathrm{c}}=0.015$. The slopes differ significantly, DEDICA and MBE find the strongest relation with $a=0.090$ and $a=0.103$ respectively, DTFE and kNN follow with $a=0.081$ and $a=0.075$, all with $\sigma_{a}=0.014-0.015$.

There appears to be a significant dip at high densities (at $0.9 \sigma, \hat{\rho}\left(\boldsymbol{r}_{\boldsymbol{i}}\right)=0.045 \mathrm{gal} \mathrm{Mpc}^{-3}$ ) in the color-density relation for the MBE-inferred densities. The cause of this dip is unclear, but could conceivably be due to a morphological or color transition at the edge of clusters in this sample (see, e.g., van Dokkum et al. 1998; Braglia et al. 2007, for more direct evidence of such transitions).

\subsubsection{The color-concentration-density relation}

There exists also a correlation between the structure of galaxies and their environment (e.g., Dressler 1980; Driver et al. 2006); by combining the color-density and color-structure relations together, an even clearer bimodality in galaxy properties can be found (Baldry et al. 2006). Here we use the inverse concentration $i C$ as a tracer of a galaxy's structure, following Baldry et al. (2006). We show the color-inverse concentration relations for six bins in standardized density for the "shell" sample in Fig. $10^{4}$. In

4 We note that these figures are not directly comparable with, say, Fig. 10 of Baldry et al. (2006), for two reasons: (1) the densities used 

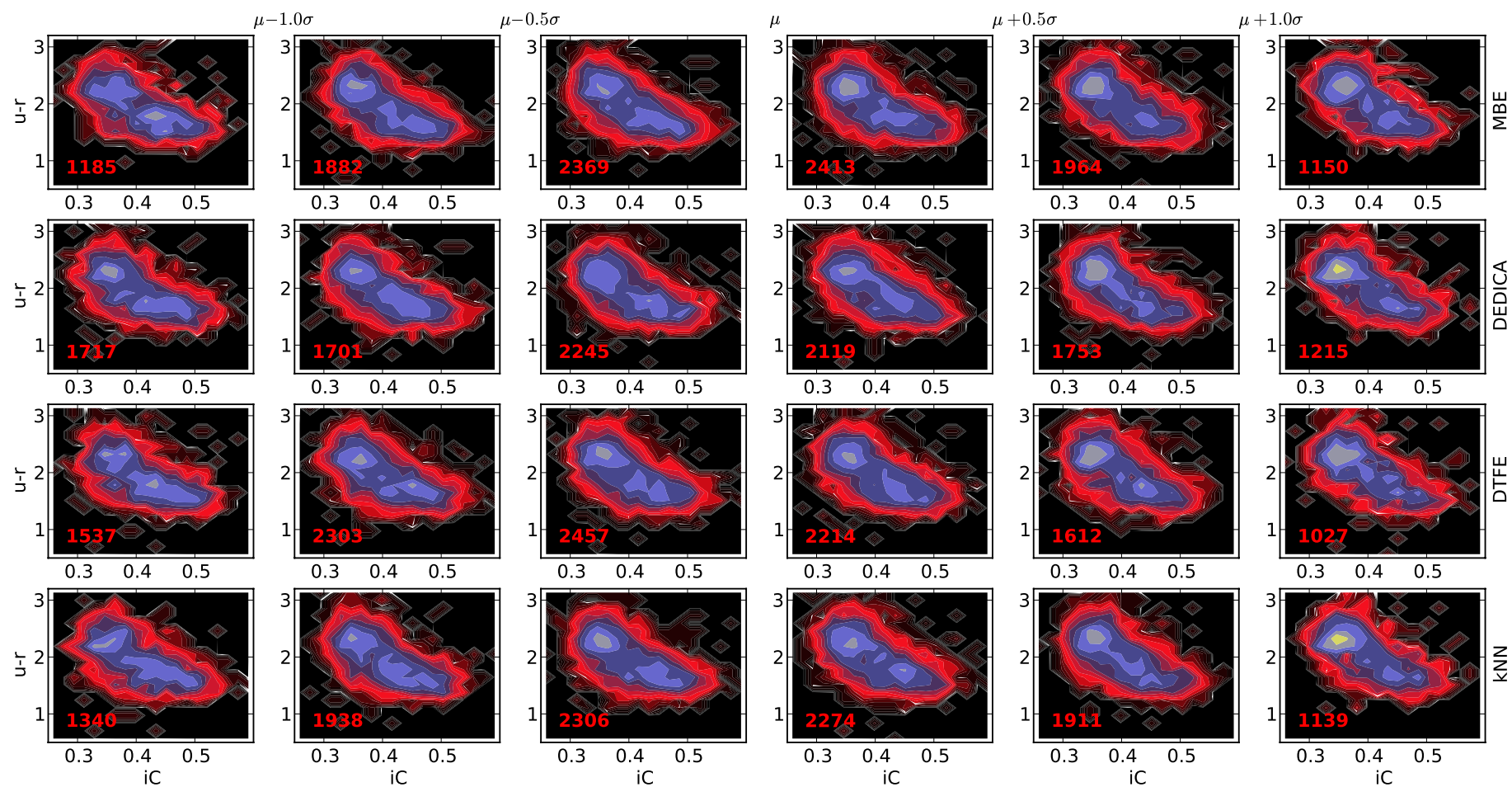

Fig. 10. Normalized contour plots in color-concentration space for six bins in standardized density, for the SDSS "shell" sample and for each of the four density estimators. The subfigures are cropped to the same color and concentration range as Baldry et al. (2006). To aid comparison, every subfigure uses the same color levels. The red number in the lower left corner shows the number of galaxies in the bin.

all density bins (and for each density estimator) a well-defined red, concentrated (small $i C$ ) peak and a blue, extended (large $i C$ ) clump can be seen; but the contrast between these features varies with density as expected.

For all methods, the figures in the first and last column of Fig. 10 indicate that the blue, extended clump is more pronounced in the lowest density regions and that the red, concentrated galaxies are more common in the highest density regions. However, the figures in the inner four columns show a clear transition from the first column to the last for MBE, but hardly for DTFE, with DEDICA and kNN in between. Therefore, MBE differentiates the two classes of galaxies in the intermediate density regions better.

\section{Conclusions and recommendations}

All four methods are applicable in astronomical problems; overall we prefer the modified Breiman estimator. For the artificial datasets the kernel based methods outperform the DTFE and $\mathrm{kNN}$ with respect to the integrated square error and Kullback-Leibler divergence. The correct kernel size determination is a crucial factor, and DEDICA fails to estimate the kernel size correctly in more complex datasets such as the Millennium simulation and SDSS.

\subsection{Artificial and simulated datasets}

From our artificial datasets we conclude that the adaptivekernel-based methods, MBE and DEDICA, are better at recovering the "true" density distributions than the $\mathrm{kNN}$ or DTFE methods. However, DEDICA clearly has difficulties with

for the binning are three-dimensional, standardized densities, not twodimensional surface galaxy densities; (2) we consider different mass ranges. spatially-complex distributions, making it unsuitable for use on problems related to the large-scale structure of the Universe (see Sect. 5.3).

All methods overestimate the density of dense regions, with DTFE having the highest deviation from the true density because the DTFE density approaches infinity if the volume of the surrounding tetrahedra approaches zero. On the other hand, all methods almost equally underestimate the density in low density regions.

The DTFE even produces zero densities for points on the convex hull of the dataset. However, in an astronomical setting, this is not always problematic. The convex hull represents the edge of the sample: physically there are galaxies beyond the edge which are not represented in our estimated densities. Therefore all methods produce densities that are lower than the unknown "true" densities in these regions. The zero values of the DTFE density estimator can be used as an implicit indicator that the density estimation was not successful for these galaxies. With the other methods, these galaxies silently end up in a too-low density bin.

Pelupessy et al. (2003) have performed a similar comparison of a kernel-based method (using a spline kernel with a window size of 40 nearest neighbors) with DTFE, with the true density being unknown. They found that in dense regions the kernelbased method yields lower densities than DTFE. However, they also mentioned that the performance of the kernel-based method varies with the choice of kernel and smoothing parameter. DTFE indeed performs better than the kernel-based method in producing a high-resolution density field with highly detailed structure.

\subsection{SDSS datasets}

From the SDSS datasets we conclude that although the estimators produce different distributions of densities, they all give 
Table 3. Computational complexity and memory requirement of density estimation methods.

\begin{tabular}{llll}
\hline \hline Method & \multicolumn{1}{c}{ Computational complexity } & Memory requirement & \multicolumn{1}{c}{ Comments } \\
\hline kNN & straightforward: $O\left(d N^{2}\right)$ & $N d$ & kd-tree inefficient when $d \gg 1$ \\
& using kd-tree : $O(d N \log N)$ & & \\
DTFE & $O(N \log N)$ & $N$ & Available implementation only for $d=3$ \\
MBE & $O(d N)$ & $G^{d}$ & Inefficient with memory when $d>3$ \\
DEDICA & $O\left(d N^{2}\right)$ & $d N$ & Computationally inefficient \\
\hline
\end{tabular}

Notes. $N=$ number of data points, $d=$ dimension, $G=$ number of grid points. DTFE numbers for $d=3$ only.

results in analysis that are consistent with the literature. While the densities produced by DEDICA are inconsistent with the expected average field density, they can still be used in standardized form.

The kNN and DTFE are very sensitive to local perturbations, producing high densities in overall low density environments. This places the more uniform distributed blue galaxies in higher density bins and broadens the distribution of densities. Therefore it is more difficult to appreciate the effect of density, e.g., in the relation with color and concentration, for DTFE and $\mathrm{kNN}$ than for MBE and DEDICA. Furthermore, the kNN method overestimates the average field density. We attribute this to the fact that $\mathrm{kNN}$ does not produce normalized fields.

For kernel based methods it is crucial to select a good kernel size. From our experience we conclude that it is difficult to define a one-size-fits-all initial kernel size algorithm.

The MBE indicates a peculiarity in the color distribution of galaxies at intermediate densities. This could be an indication of evolution of galaxies at the edge of galaxy clusters that could not be detected with the other methods.

\subsection{Dedica}

Although DEDICA performs very well for most simulated datasets, it performs badly for the simulated dataset 4 (Figs. 5, 6) and the astronomical datasets (Fig. 7).

We attribute the failure of DEDICA in these cases to the behaviour of the cross validation for inhomogeneously distributed data. As we already indicated in Sect. 2.3, DEDICA aims for a globally optimal result, instead of performing a locally adaptive optimization of kernel widths. This may result in low performance in cases where the underlying distribution consists of two quite different components, as is the case for the simulated dataset 4.

For the astronomical data, DEDICA produces kernels with very small sizes. As an example, we compare the optimal window widths for dataset MSG-DTFE as found by DEDICA and MBE, respectively, see Fig. 11. It is very clear that DEDICA has optimal kernel sizes which are much smaller than those of MBE. In this case, the data are highly clustered and the underlying density distribution is very non-smooth. Probably, the millennium density has a non-differentiable, fractal-like nature, which violates the basic assumption of kernel density estimators that the underlying density should be continuous, differentiable, and bounded. For MBE this has less serious consequences, as it only computes a pilot estimate once, instead of trying to optimize the window widths iteratively.
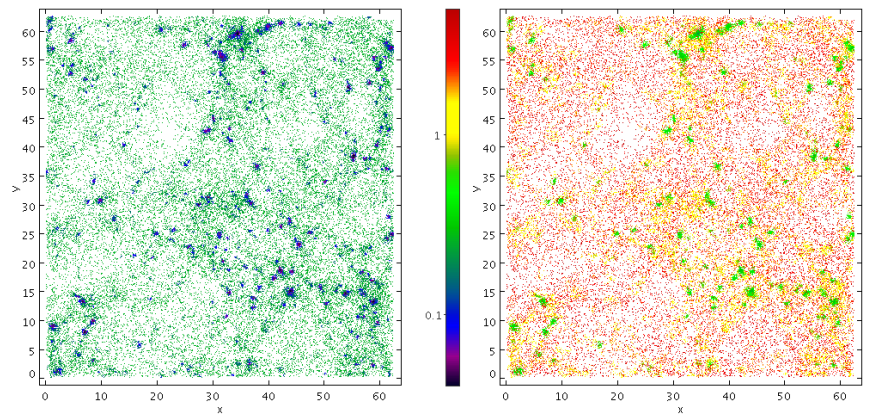

Fig. 11. Optimal window sizes (showing color in log-scale) for dataset MSG-DTFE produced by DEDICA (left) and MBE (right).

\subsection{Computational complexities}

In Table 3 we present a summary of the computational complexities and memory requirements of the various density estimation methods. MBE is the most efficient (linear complexity), DTFE and an efficient kNN implementation using kd-trees have slightly higher complexity, while DEDICA has quadratic complexity. Regarding memory usage, MBE has the advantage that its memory requirement only depends on the number of grid points, but it does not scale well with increasing number of dimensions.

\subsection{Recommendations}

Each method has its own strengths, therefore the choice of method may vary depending on the problem at hand. For example, having a proper point density is important when studying the relationships between properties of individual galaxies and their environment, while a high resolution density field is more important when studying the large scale structure of the universe.

In this paper we focus on point densities and we conclude that MBE is our preferred density estimator. It produces densities that are consistent with expectations from literature and provides more discriminating power than the other methods. Furthermore it is the fastest method of our tests. A drawback is that a good determination of the initial kernel size is non trivial. We recommend an interactive process.

The other kernel method, DEDICA, fails to produce correct densities for our astronomical datasets. Furthermore it is the slowest of the tested methods. Therefore we cannot recommend DEDICA, at least not for highly clustered data.

The DTFE produces overall good densities, but is very sensitive to local effects. It produces small regions of large densities, even in otherwise low density regions. The computational complexity puts an upper limit on the number of sources to include, even though very fast implementations exist. However, 
the DTFE is better in discovering shapes in the density fields than the kernel based methods, such as determining the filamentary structure of the cosmic web.

The kNN method, one of the most used density estimators in astronomy, performs rather badly in our tests. It does not produce normalized density fields, which results in overestimated densities. The kNN is very sensitive to local effects which broadens the density distribution. At the same time it produces non-zero densities in regions far away from any sources. The positive side of $\mathrm{kNN}$ is that it can be implemented quickly in a few of lines of code. This makes the kNN an attractive choice for quick and dirty density estimations, but we recommend that it should not be used for more serious density estimation.

Acknowledgements. We would like to thank our collaborators in the AstroVIS project, Prof. Dr. J.M. van der Hulst, Prof. Dr. E. Valentijn, and Prof. Dr. A. Helmi, for their continued advice and support during the gestation of this work. We thank Prof. A. Pisani for allowing us to use his DEDICA code for our work and Dr. E. Platen for the use of his DTFE code. The comments of our referee, Dr. Stéphane Colombi, helped greatly to improve the presentation. This research is part of the project "Astrovis", research program STARE (STAR E-Science), funded by the Dutch National Science Foundation (NWO), project No. 643.200.501

\section{Appendix A: Mock samples - selection effects}

In order to study the impact of selection effects on the density estimations of SDSS galaxies - in particular for the "cone" sample - we created four mock samples. The densities produced for these mocks are compared to our "cone" sample. The cone sample represents a region of SDSS that is $49 \%$ more dense than average. To compare with this overdensity, the mock samples were created with the same average field density of $\left\langle\rho_{\text {cone }}(\boldsymbol{r})\right\rangle=$ 0.0196 which corresponds to 4024 sources.

We distinguish five different effects that we want to investigate. Any difference of the results of the density estimators that is not explained by the points below are attributed to effects intrinsic to the "cone" sample.

1. Background differences of the estimators. A uniform box with of size $58.9 \mathrm{Mpc}$ with an average density of $\left\langle\rho_{\text {cone }}(\boldsymbol{r})\right\rangle=$ 0.0196 is created ("Mock Sample A", 4020 sources).

2. Effects of the conical shape of the "cone" sample. A sample with the same average density but with the shape of our "cone" sample is created ("Mock Sample B", 4010 sources).

3. Effects of the luminosity selection. Using the derived selection function, sources are removed from Mock Sample B in such a way that the radial distribution of sources represents the radial distribution of the "cone" sample ("Mock Sample C", 1027 sources). This is done by assigning to every mock source a uniform random number between 0 and 1 and removing all sources where this number is larger than the value of the selection function at that distance.

4. Effects of clustering of the sources. A sample of 49287 galaxies with the same angular shape as Sample B is selected from the L-Galaxies of the full Millennium Simulation. A distance and magnitude limit is imposed to select 4024 galaxies with the same shape as the "cone" sample ("Millennium Mock Sample").

5. Edge effects. Sources at the edges will have underestimated densities. To study this effect we removed about $30 \%$ of sources that are closest to the edge in our mock samples.

The radial distributions of the samples are shown in Fig. A.1. The corresponding density distributions of all the points are

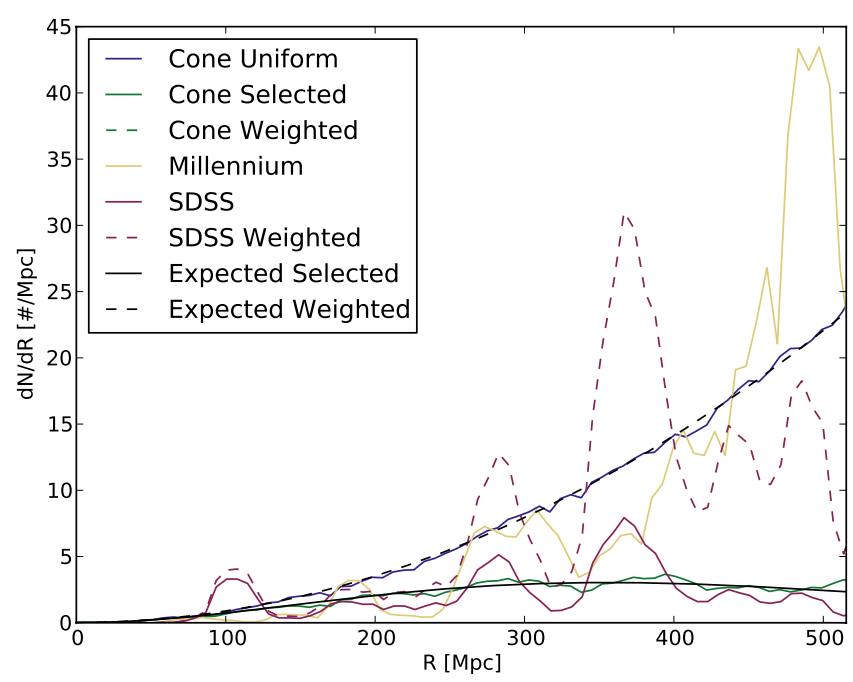

Fig. A.1. Radial distribution of the mock samples. The dashed black line shows the expected distribution of the galaxies, the black solid line after applying a luminosity selection. The (red) distribution of the "cone" sample shows more structure than a uniform mock would have (green), due to internal clustering.

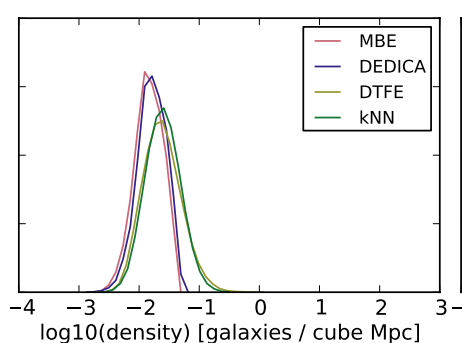

Sample A

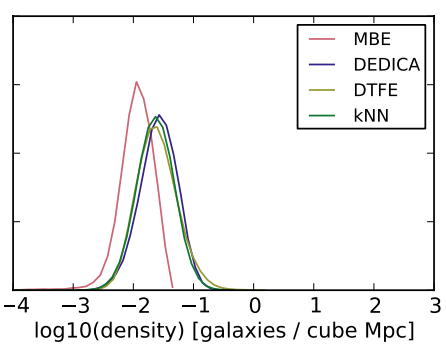

Sample B

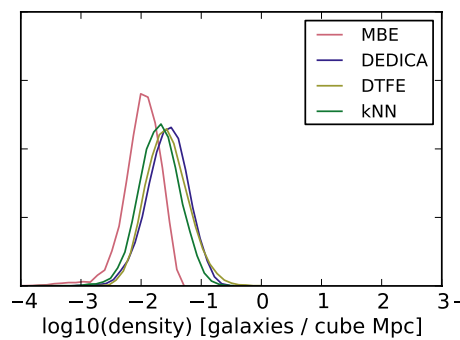

Sample C

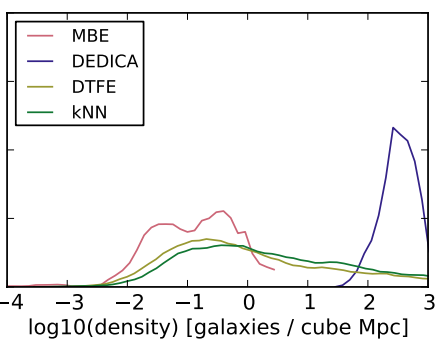

Millennium

Fig. A.2. Normalized density distributions from the four density estimators for the four mock samples (A, B, C, and Millennium), all with the same average density $\left(\hat{\rho}(\boldsymbol{r})=0.0196 \mathrm{gal} \mathrm{Mpc}^{-3}\right)$.

plotted in Fig. A.2 and without the edge points in Fig. A.3. In the uniform box (Sample A), the density distributions of kNN and DTFE are very similar (except for the high-end DTFE tail). The cone shape only has a significant effect on the kernel based methods, DEDICA producing slightly higher densities and MBE slightly lower. When simulating and correcting for a luminosity selection (Sample C), the distributions change only slightly, justifying the $90 \%$ incompleteness we allow. The MBE and kNN distributions look very similar, as do the DEDICA and DTFE distributions. From the Millennium Mock Sample, it is clear that the clustering of the sources has a large effect on the estimated densities. The densities estimated by DEDICA are several orders of magnitude higher than the estimations of the other methods. This overestimation correlates with the small kernel sizes used 


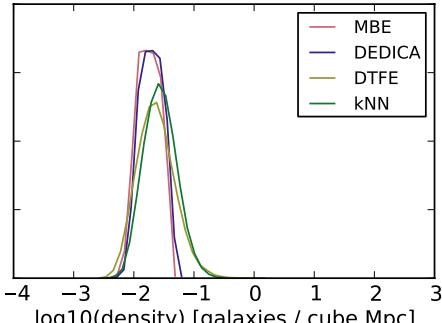

Sample A

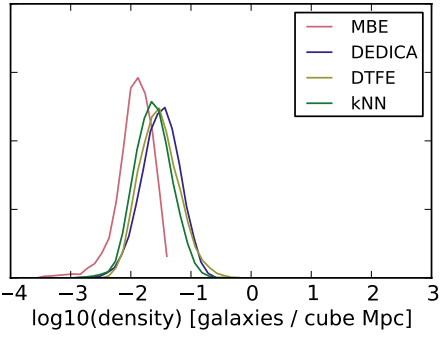

Sample C

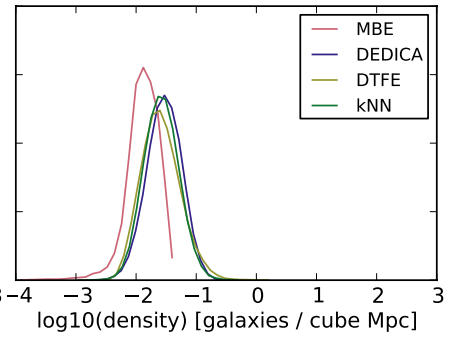

Sample B

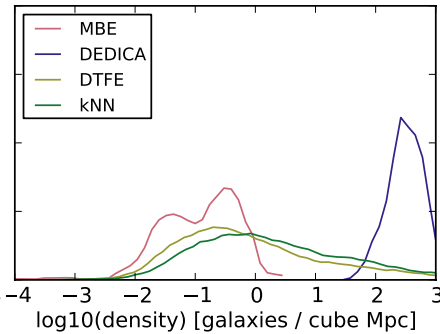

Millennium
Fig. A.3. As in Fig. A.2, but now with approximately $30 \%$ of data closest to the edges of each sample removed.

by DEDICA, as discussed in Sect. 5.3. There is also an apparent bimodality visible in the MBE density distribution.

\section{A.1. Edge effects}

By comparing Fig. A.2 with A.3, it is possible to study the effect of edges on the density distributions. In Fig. A.3 30\% of the points closest to the sample edges are removed. In all methods, the lower density bins are overrepresented in Fig. A.2 due to edge effects but in Fig. A.3 the low-end tails are still visible. Any edge effect on the tails therefore must be minor.

\section{Appendix B: DTFE Monte-Carlo sampling}

A modified, but equivalent, version of the sampling procedure described in Sect. 3.2.1 is used for the Monte Carlo sampling of the DTFE field. Due to the high sensitivity of DTFE to shot noise, the estimated field will contain very small regions with very high density: the maximum estimated density for the milliMillennium dataset is more than 10000 times higher than the average density. Following the exact procedure as with MBE will result in more than 10000 randomly chosen points to be discarded for every accepted point, slowing the procedure significantly.

This can be alleviated by lowering the maximum possible value for $p$ in regions with a low density. The height of this maximum can vary as function of location without affecting the Monte Carlo simulation, as long as it is always above or equal to the true density $P$ and all the points $(x, y, z, p)$ are drawn uniformly below it. The maximum density for a field location is set to the maximum density $D_{\mathrm{c}}$ of the Delauney cell $c$ at that location. Figure B. 1 shows the maximum density value for a one dimensional example as a dotted red line.

Step 2a of Sect. 3.2.1 is modified to ensure a uniformly drawn sample. First a Delaunay cell (simplex) $c$ is selected, and then a point $r$ within the simplex with a test density $p$ below $D_{\text {c }}$. More points should be drawn from larger cells and from cells with a higher density in order to get a uniform selection. This is
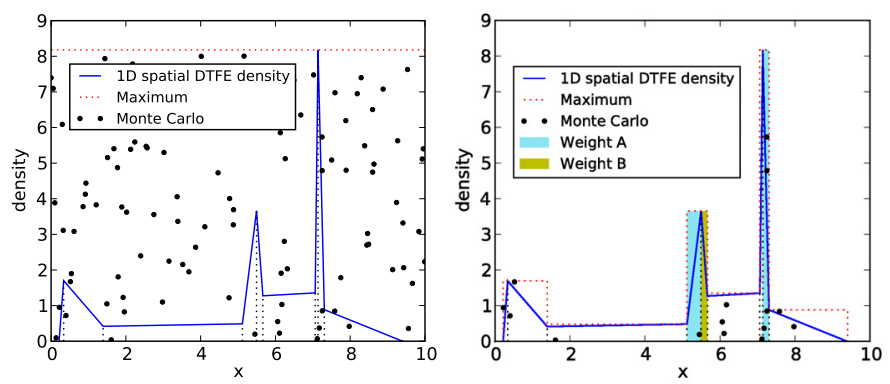

Fig. B.1. The original (left) and modified (right) Monte Carlo process for a 1 dimensional sample. The vertices on the blue line are the original sample points, the blue line itself the DTFE field. Black points are uniformly drawn in the area below the red line, those below the blue line are included in the new sample. In this 1D example, the lowering of the red line gives a factor 6 improvement in performance, in our MSG dataset this is more than a factor 10000 . The weights are given by the colored areas: the cyan cells have the same weight. The yellow cell has the same maximum density or volume as one of the cyan cells but a lower weight. (Densities are linear spatial densities, not probability densities).

achieved by giving these cells a higher preference when selecting a random cell. This preference is quantified by a weight $w$ and uniformity is ensured by choosing $w=V_{\mathrm{c}} D_{\mathrm{c}}$, because this is exactly the $d+1$-dimensional volume below the red line that corresponds to that cell.

The cells are simplices (tetrahedra when $d=3$ ) with $d+1$ vertices $v_{0}$ to $v_{\mathrm{d}}$. A random position $r_{i}$ within the cell is selected by choosing $d$ uniform random numbers $a_{j}$ between 0 and 1 as

$r_{i}=v_{0}+\sum_{j=1}^{d} a_{j}\left(v_{j}-v_{0}\right)$,

keeping only the $1 / d$ ! points actually within the cell. The random density $p$ is selected uniformly between 0 and $D_{\text {c }}$. A new cell is selected if the test density $p$ is higher than the interpolated "true" density $P$ at the location $r_{i}$.

\section{References}

Abazajian, K. N., Adelman-McCarthy, J. K., Agüeros, M. A., et al. 2009, ApJS, 182,543

Adelman-McCarthy, J. K., Agüeros, M. A., Allam, S. S., et al. 2007, ApJS, 172, 634

Aragón-Calvo, M. A., van de Weygaert, R., \& Jones, B. J. T. 2010, MNRAS, 408, 2163

Baldry, I., Balogh, M. L., Bower, R., et al. 2006, MNRAS, 373, 469

Ball, N., Loveday, J., \& Brunner, R. J. 2008, MNRAS, 383, 907

Balogh, M. L., Baldry, I. K., Nichol, R., et al. 2004, ApJ, 615, L101

Blanton, M. R., \& Roweis, S. 2007, AJ, 133, 734

Braglia, F., Pierini, D., \& Böhringer, H. 2007, A\&A, 470, 425

Breiman, L., Meisel, W., \& Purcell, E. 1977, Technometrics, 19, 135

Coles, P., \& Jones, B. 1991, MNRAS, 248, 1

Cowan, N. B., \& Ivezic, Z. 2008, ApJ, 674, 13

Csiszar, I. 1991, Ann. Statis., 19, 2032

De Lucia, G., \& Blaizot, J. 2007, MNRAS, 375, 2

Deng, X., He, J., \& Wen, X. 2009, ApJ, 693, 71

Dressler, A. 1980, ApJ, 236, 351

Driver, S. P., Allen, P. D., Graham, A. W., et al. 2006, MNRAS, 368, 414

Eguchi, S., \& Copas, J. 2006, J. Multivar. Anal., 97, 2034

Epanechnikov, V. A. 1969, Theor. Probab. Appl., 14, 153

Felten, J. E. 1977, AJ, 82, 861

Gingold, R., \& Monaghan, J. 1977, MNRAS, 181, 375

Helmi, A., \& de Zeeuw, P. T. 2000, MNRAS, 319, 657 
A\&A 531, A114 (2011)

Hubble, E., \& Humason, M. L. 1931, ApJ, 74, 43

Jasche, J., Kitaura, F. S., Wandelt, B. D., \& Enßlin, T. A. 2010, MNRAS, 406, 60

Knuth, D. E. 1981, The Art of Computer Programming: Seminumerical Algorithms, 2nd edn. (Addision-Wesley)

Loader, C. L. 1999, Ann. Stat., 27, 415

Lucy, L. B. 1977, AJ, 82, 1013

Maciejewski, M., Colombi, S., Springel, V., Alard, C., \& Bouchet, F. R. 2009, MNRAS, 396, 1329

Martínez, V. J., \& Saar, E. 2002, Statistics of the Galaxy Distribution (Chapman \& Hall/CRC)

Okabe, A., Boots, B., Sugihara, K., \& Chiu, S. 2000, Spatial Tessellations (Chichester: John Wiley)

Park, B. U., \& Marron, J. S. 1990, J. Am. Stat. Assoc., 85, 66

Parzen, E. 1962, Ann. Math. Stat., 33, 1065

Pelupessy, F., Schaap, W., \& van de Weygaert, R. 2003, A\&A, 403, 389
Pisani, A. 1993, MNRAS, 265, 706

Pisani, A. 1996, MNRAS, 278, 697

Romano-Díaz, E., \& van de Weygaert, R. 2007, MNRAS, 382, 2

Ruppert, D., Sheather, S. J., \& Wand, M. P. 1995, J. Am. Statis. Assoc., 90, 1257 Schaap, W., \& van de Weygaert, R. 2000, A\&A, 363, 29

Schechter, P. 1976, ApJ, 203, 297

Sheather, S. J. 1992, Comput. Stat., 7, 225

Silverman, B. W. 1986, Density Estimation for Statistics and Data Analysis (Chapman and Hall)

Sousbie, T., Colombi, S., \& Pichon, C. 2009, MNRAS, 393, 457

Springel, V., White, S. D. M., Jenkins, A., et al. 2005, Nature, 435, 629

Strateva, I., Ivezić, Ž., Knapp, G. R., et al. 2001, AJ, 122, 1861

van Dokkum, P. G., Franx, M., Kelson, D. D., et al. 1998, ApJ, 500, 714

Wilkinson, M. H. F., \& Meijer, B. C. 1995, Comp. Meth. Prog. Biomedicine, 47, 35

York, D. G., Adelman, J., Anderson, Jr., J. E., et al. 2000, AJ, 120, 1579 